\title{
Outer automorphism groups
}

\author{
Dissertation \\ zur Erlangung des Akademischen Grades eines \\ Doktors der Naturwissenschaften
}

von

\section{Gábor Braun}

\author{
geboren in Budapest, Ungarn
}

vorgelegt beim Fachbereich Mathematik

der Universität Duisburg-Essen, Standort

Essen

Essen 2003 
Vorlage der Dissertation: 10.3.2003

Tag der Disputation: $\quad$ 29.4.2003

\section{Prüfungsausschuss:}

Vorsitzender:

PD Dr. Wolfgang Lempken

Gutachter:

Prof. Dr. Rüdiger Göbel

Prof. Dr. Otto Kegel 


\section{Acknowledgments}

I am grateful to my supervisor Rüdiger Göbel for inviting me to Essen to write my Phd thesis. I would like to thank Simone Wallutis for her great help to formulate my thoughts. 


\section{Contents}

$\begin{array}{ll}\text { Notations } & 5\end{array}$

\begin{tabular}{lll}
\hline 1 & Introduction & 7
\end{tabular}

1.1 Outer homomorphisms . . . . . . . . . . . . . . . . . . 7

1.2 Abstract . . . . . . . . . . . . . . . . . . . . . . . . . 8

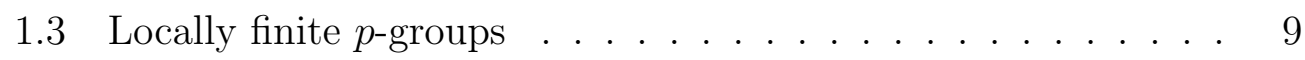

1.4 Other classes of groups $\ldots \ldots \ldots 11$

1.5 Methods of construction . . . . . . . . . . . . . . . . . . . 12

Set theory . . . . . . . . . . . . . . . . . . 12

Structure of the constructed groups . . . . . . . . . . . 13

\begin{tabular}{|lr}
2 & Complete groups under CH \\
\hline
\end{tabular}

2.1 Background and main results . . . . . . . . . . . . . . 15

2.2 The construction . . . . . . . . . . . . . . . . . . . . . . . 16

2.3 The choice of almost disjoint sets $A_{\beta} \ldots \ldots \ldots \ldots$. . . . . 24

2.4 Characterization of outer automorphisms . . . . . . . . . . . . 28

$\begin{array}{lll}3 & \text { Large } p \text {-groups } & 31\end{array}$

3.1 Preliminaries and the main theorem . . . . . . . . . . . . . 31

3.2 Construction of monoids . . . . . . . . . . . . . . . . . . . 34

3.3 A fully rigid system of abelian $p$-groups . . . . . . . . . . . . 36

Preliminaries . . . . . . . . . . . . . . . 37

The fully rigid system . . . . . . . . . . . . . . 38

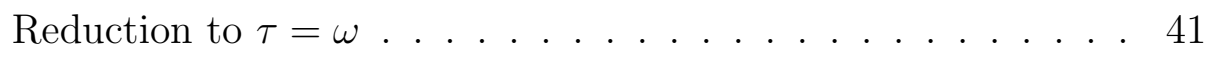

The Black Box . . . . . . . . . . . . . . . . . . . . . . 42 
Construction of abelian $p$-groups . . . . . . . . . . . . . 44

3.4 Construction of locally finite $p$-groups . . . . . . . . . . . . 56

Initial step . . . . . . . . . . . . . . . . . . . 57

The monoids $M_{X}^{\alpha}$ s $\ldots \ldots \ldots$. . . . . . . . . . 57

Limit step . . . . . . . . . . . . . . . . . . . . . . . . . . . . . 58

Successor step . . . . . . . . . . . . . . . . . . . . . . 59

Determining $\operatorname{Emb}\left(G_{X}, G_{Y}\right) \ldots \ldots \ldots \ldots$. . . . . . . 64 


\section{Notations}

\section{Groups}

$C(n) \quad$ cyclic group of order $n$

$Q^{x} \quad=x^{-1} Q x$, conjugate of $Q$

$a^{b} \quad=b^{-1} a b$, conjugate of $a$

$a^{*} \quad b a^{*}=b^{a}$, inner automorphism generated by $a$

$C_{G}(P) \quad$ centralizer of $P$ in $G$

$[G: H]$ index of the subgroup $H$ in $G$

$A \curlywedge B \quad=\bigoplus_{b \in B} A e_{b} \rtimes B$, restricted wreath product of $A$ and $B$

$\mathbb{Z} Q \quad$ group ring of $Q$ with integer coefficients

\section{Set theory}

$\mathbb{N} \quad=\{0,1, \ldots\}$, the set of natural numbers

$H \backslash G$ set difference of $H$ and $G$

$|S| \quad$ cardinality of $S$

$1 \quad$ identity mapping or unit element

$\operatorname{Br} T \quad$ set of branches of the tree $T$

${ }^{<\omega} \alpha \quad$ tree of all functions $n \rightarrow \alpha$ for $n \in \omega$

$l(\sigma) \quad$ height of $\sigma$ in a tree

\section{Monoids}

$b^{m} \quad$ the action of $m$ on $b$

$M / B \quad$ factor of $M$ by $B$, see Definition 3.1 .1

$F \rtimes G$ semi-direct product of the semigroups $F$ and $G$ 


\section{Abelian groups}

$\begin{array}{ll}\hat{A} & p \text {-adic completion of } A \\ \bar{A} & \text { torsion-completion of } A \\ J_{p} & =\hat{\mathbb{Z}}, \text { ring of } p \text {-adic integers } \\ m A & \text { subgroup of elements divisible by } m \\ A[n] & \text { subgroup of elements bounded by } n \\ m A[n] & =(m A)[n], \text { subgroup of elements of } m A \text { bounded by } n \\ G^{(X)} & \text { direct sum of copies of } G, \text { one copy for each } x \in X \\ \operatorname{Hom}(A, B) & \text { set of homomorphisms from } A \text { to } B \\ \operatorname{Small}(A, B) & \text { set of small homomorphisms from } A \text { to } B \\ B \subseteq \subseteq_{*} A & B \text { is a pure subgroup of } A\end{array}$

\section{Homomorphisms}

$\begin{array}{ll}\text { Aut } G & \text { automorphism group of } G \\ \text { Inn } G & \text { inner automorphism group of } G \\ \operatorname{Emb}\left(G_{1}, G_{2}\right) & \text { set of embeddings of } G_{1} \text { into } G_{2} \\ \operatorname{Emb} G & =\operatorname{Emb}(G, G), \text { monoid of self-embeddings of } G\end{array}$




\section{Chapter 1}

\section{Introduction}

In this thesis, we consider outer embeddings of locally finite $p$-groups. Here, in this chapter, we summarize known and also the newly developed results. We also formulate a conjecture for future research.

We begin with a short discussion of outer homomorphisms before we give a brief outline of this thesis.

\subsection{Outer homomorphisms}

It is well known that a non-trivial cyclic group of odd order cannot occur as the automorphism group of a group. It is also known that a group has a trivial automorphism group exactly when it contains at most two elements. Part of the reason for both facts is the existence of inner automorphisms.

Recall that every group $G$ can be mapped to its automorphism group Aut $G$ by

$$
*: G \rightarrow \operatorname{Aut} G, \quad(h) g^{*}=g^{-1} h g .
$$

The kernel of $*$ is the centre, $Z(G)$, of $G$, the image of $*$ is the inner automorphism group, Inn $G$, of $G$ and the cokernel of $*$ is the outer automorphism group, Out $G:=\operatorname{Aut} G / \operatorname{Inn} G$, of $G$.

Thus the idea behind the outer automorphism group is to get rid of the unavoidable automorphisms. So, it is a natural question whether every group 
appears as an outer automorphism group. Indeed, an affirmative answer was given in [19]. In fact, even more is known: every group appears as outer automorphism group of a metabelian group (see [11, 10, 9]) and a simple group (see [3]).

In generalization of the above, one can examine representation of monoids as endomorphism monoids of groups. Again, because of the existence of inner automorphisms, it seems convenient to use outer endomorphisms, which we define below. If we want to construct a system of groups with prescribed homomorphisms between them, then a convenient choice will be the outer homomorphisms.

If $G_{1}$ and $G_{2}$ are two groups, we define a congruence $\sim$ on $\operatorname{Hom}\left(G_{1}, G_{2}\right)$, the set of homomorphisms between $G_{1}$ and $G_{2}$ by

$$
a \sim b \Longleftrightarrow \exists g \in G_{2}: a=b \cdot g^{*} \quad\left(a, b \in \operatorname{Hom}\left(G_{1}, G_{2}\right)\right)
$$

and then define the set of outer homomorphisms between $G_{1}$ and $G_{2}$ as

$$
\operatorname{OutHom}\left(G_{1}, G_{2}\right):=\operatorname{Hom}\left(G_{1}, G_{2}\right) / \sim
$$

Note that $\sim$ is compatible with composition of homomorphisms, so composition of outer homomorphisms make sense. In particular, if $G_{1}=G_{2}=G$ then OutEnd $G:=\operatorname{OutHom}(G, G)=\operatorname{End} G / \sim$ is a monoid, which we call the outer endomorphism monoid of $G$.

In this thesis, however, we shall restrict to the automorphisms and the embeddings (injective endomorphisms) of $G$ : we denote by $\operatorname{Emb} G$ the monoid of embeddings of $G$ and define OutEmb $G:=\operatorname{Emb} G / \sim$ to be the outer embedding monoid of $G$. This is clearly a submonoid of OutEnd $G$.

\section{$1.2 \quad$ Abstract}

In this thesis, we consider outer automorphisms and embeddings of locally finite $p$-groups.

Recall that a group is locally finite if every finitely generated subgroup 
is finite. A group is complete if both its centre and its outer automorphism group are trivial. In other words, $G$ is complete if it is canonically (i.e. via * from (1.1) ) isomorphic to its automorphism group.

In Chapter 2, we show (Theorem 2.1.1) that there is a complete locally finite $p$-group of cardinality $\aleph_{1}$ provided the continuum hypothesis $2^{\aleph_{0}}=\aleph_{1}$ holds. This is a refinement of Thomas' construction (see [25]), which works under a stronger set theoretic assumption, namely, the diamond principle $\diamond_{\omega_{1}}$. Note that $\diamond_{\omega_{1}}$ follows from Gödel's constructibility axiom. Our construction is also more elementary as it avoids the theory of universal locally finite $p$-groups.

In Chapter 3, we characterize those monoids $M$ which appear as outer endomorphism monoid of a locally finite $p$-group with trivial centre. Moreover, for every such $M$, there is a fully rigid system $\left(G_{X}: X \subseteq \kappa\right)$ of locally finite $p$-groups with OutEmb $G_{X} \cong M$ and $\left|G_{X}\right|=\kappa$ for all $X \subseteq \kappa$. By fully rigidity we mean that, for every $X, Y \subseteq \kappa$, if $X \nsubseteq Y$ then $G_{X}$ is not embeddable into $G_{Y}$, but if $X \subseteq Y$ then the embeddings of $G_{X}$ into $G_{Y}$ are precisely the compositions of the natural inclusion $G_{X} \subseteq G_{Y}$ and a self-embedding of $G_{Y}$. Informally, this means that the embeddings are just the unavoidable ones.

There are some technical restrictions on the cardinal $\kappa$, the most important ones are $\kappa>2^{\aleph_{0}}, \kappa>|M|$ and cf $\kappa>\omega$. We conjecture that the result above is also true for every $\kappa$ for which $\kappa \geq|M|$ and $\kappa \geq \aleph_{1}$.

We also characterize the monoids which appear as outer endomorphism monoid of a group as those in which every left or right invertible element is invertible.

Our conjecture is that, for every monoid $M$ and cardinal $\kappa \geq|M|$. $\aleph_{1}$, there is a locally finite $p$-group $G$ of cardinality $\kappa$ such that the outer endomorphism monoid of $G$ is isomorphic to $M$. Probably there is also a fully rigid system of such groups as described above.

\subsection{Locally finite $p$-groups}

In this section, we give an overview of the known results about outer endomorphisms of locally finite $p$-groups. Note that the notion of outer homo- 
morphism and outer embedding have firstly been introduced in this thesis, so all the former results we mention are about outer automorphisms.

In 1966 Gaschütz showed that every finite $p$-group with at least 3 elements has a non-trivial outer automorphism (see [8]). This theorem was successfully generalized to some classes of infinite $p$-groups. Zaleskii showed that every infinite nilpotent $p$-group has a non-trivial outer automorphism (see [26]). Furthermore, in [20] Menegazzo and Stonehewer proved that the outer automorphism group of any nilpotent p-group, except some obvious ones, contains large $p$-subgroups. Puglisi showed that hypercentral $p$-groups of height $\omega$ have at least continuum many outer automorphisms. (Hypercentral groups are generalizations of nilpotent groups. Height is a generalization of the nilpotency class.)

All the groups mentioned above belong to some special classes of locally finite $p$-groups. Concerning locally finite $p$-groups in general, Puglisi showed that every countably infinite locally finite $p$-group has $2^{\aleph_{0}}$ outer automorphisms (see [22]), and thus generalized Gaschütz' theorem to countable groups. However, Thomas showed that there exists a complete locally finite $p$-group of cardinality $\aleph_{1}$, at least under the set theoretical assumption $\diamond_{\omega_{1}}$. In Chapter 2 we will present this argument in a modified form establishing the same result under a weaker and more known hypothesis, the continuum hypothesis $2^{\aleph_{0}}=\aleph_{1}$.

So, very little is known about outer homomorphisms of locally finite $p$ groups of uncountable cardinality but at most continuum. However, the case is totally different for larger groups.

Using a totally different construction, Dugas and Göbel [4] proved that there are complete locally finite $p$-groups of every cardinality $\left(\lambda^{\aleph_{0}}\right)^{+}$, without any set theoretical assumption. Furthermore, they proved that every countable locally finite $p$-group appears as an outer automorphism group of such a group.

Their method was refined in [1] by Göbel and myself showing that, for every group $H$ of cardinality at most $\lambda$, there is a locally finite $p$-group $G$ of cardinality $\left(\lambda^{\aleph_{0}}\right)^{+}$such that Out $G \cong H$. This will be further improved in Chapter 3 to show that every monoid $M$, with obvious restrictions, can ap- 
pear as an outer endomorphism monoid of a locally finite $p$-group. Moreover, there is a fully rigid system of such groups.

With regard to the results above, a natural conjecture is that for every monoid $M$ and every cardinal $\kappa \geq|M| \cdot \aleph_{1}$ there is a locally finite $p$-group $G$ with outer endomorphism monoid $M$, which we have already stated.

\subsection{Other classes of groups}

In this section we look at outer automorphisms in group classes other than locally finite $p$-groups.

Recall that torsion locally hypercentral groups decompose into their $p$ components, which are locally finite. Thus the investigation of these groups reduces to that of locally finite $p$-groups, which was the subject of the previous section.

However, in the torsion-free case, Zaleskii (see [27]) gave an example of an infinite torsion-free nilpotent group without non-trivial outer automorphisms.

We have already mentioned before that every group is an outer automorphism group of a metabelian group as shown by Göbel and Paras [9, 10, 11].

All the groups mentioned so far were either groups near to abelian or nilpotent groups or locally finite $p$-groups, all of which have many normal subgroups. On the other hand, simple groups, i.e. groups with no non-trivial proper normal subgroup, can also have arbitrary outer automorphism groups as recently shown in [3] by Droste, Giraudet and Göbel. Their method, actually, is a curiosity: They construct a circle $C$ (a combinatorial structure related to orderings) such that Aut $C$ is simple and Out Aut $C$ is a prescribed group.

There were some previous constructions of complete simple groups. Hickin (see [13]) showed the existence of $2^{\aleph_{1}}$ non-isomorphic complete universal locally finite, and hence simple, groups of cardinality $\aleph_{1}$. Assuming the generalized continuum hypothesis, Thomas generalized this result to all successor cardinals, i.e. that there exists $2^{\lambda^{+}}$non-isomorphic complete universal locally finite groups of cardinality $\lambda^{+}$. 
In fact, the construction of Hickin is a predecessor of the similar result on locally finite $p$-groups by Thomas (see [25]) already mentioned earlier.

\subsection{Methods of construction}

Here we sketch the the methods used in the following chapters to construct a locally finite $p$-group of cardinality $\aleph_{1}$ assuming the continuum hypothesis (Chapter 2) and "large" locally finite $p$-groups with prescribed outer embedding monoid (Chapter 3).

When constructing a group $G$ with outer automorphism group (or embedding monoid) $H$, we have to achieve two tasks: First, $H$ should act on $G$ by outer automorphisms (embeddings). Second, $G$ must not have other outer automorphisms.

The first task is easy. However, set theory has a strong impact on the second task. Let us illustrate this using the aforementioned result by Puglisi that every countably infinite locally finite $p$-group has uncountably many outer automorphisms. For suppose that for an infinite group $H$ we have found a locally finite $p$-group $G$ such that Out $G \cong H$. Note that $G$ must be infinite. Let us enlarge the model of set theory we are working in, e.g. by forcing, such that both $G$ and $H$ become countable in the larger model. By the Puglisi result, the outer automorphism group of $G$ will be uncountable in the larger model, and hence it will be larger than $H$.

This shows that outer automorphisms other than those in $H$ may exist but simply lie outside the model of set theory we are working in.

\section{Set theory}

The complete locally finite $p$-group of cardinality $\aleph_{1}$ in Chapter 2 is a union of a smooth increasing sequence $\left(E_{\alpha}: \alpha<\omega_{1}\right)$ of countable groups. Smooth means that, for limit ordinals $\alpha$, the group $E_{\alpha}$ is just the union of all the previous groups: $E_{\alpha}=\bigcup_{\beta<\alpha} E_{\beta}$. Mere set theory tells us that, for any automorphism $\phi$ of $E$, there are many $\alpha<\omega_{1}$ such that $E_{\alpha} \phi=E_{\alpha}$. So, all we have to do is to construct the chain $\left(E_{\alpha}: \alpha<\omega_{1}\right)$ such that no 
automorphism of $E_{\alpha}$ extends to an outer automorphism of $E$. In the original construction by Thomas, $E_{\alpha+1}$ was so chosen that a specific automorphism $f_{\alpha}$ of $E_{\alpha}$ did not extend to an outer automorphism of $E$. This required to choose the $f_{\alpha}$ 's in such a way that every automorphism of $E$ induces $f_{\alpha}$ on $E_{\alpha}$ for some $\alpha<\omega_{1}$. The diamond principle $\nabla_{\omega_{1}}$ is such an assumption, which states that this kind of functions exist.

Our improvement is that we can choose $E_{\alpha+1}$ to kill an automorphism of $E_{\beta}$ for any $\beta \leq \alpha$ and not just for $\beta=\alpha$. Now, $E_{\beta}$ has $2^{\aleph_{0}}$ automorphisms but we can kill only $\aleph_{1}$ of them, therefore we need the continuum hypothesis.

Now let us turn to the construction of large locally finite $p$-groups in Chapter 3. As in the $\aleph_{1}$ case, these groups are also unions of smooth chains of groups such that many subgroups of the chain are mapped to itself by every embedding for set-theoretical reasons. The building blocks of the groups are abelian $p$-groups with few homomorphisms among them. These groups are constructed using the classical Black Box, a combinatorial and set theoretical tool invented by Shelah.

The idea of the Black Box is that unwanted homomorphisms can be recognized by looking at their restrictions on countable subgroups. So we consider the family of all homomorphisms from countable subgroups and make sure that the "bad" ones among them do not extend. The size of the family is the same as the group we construct, so the homomorphisms can be killed one by one.

\section{Structure of the constructed groups}

Of course, set theory cannot do all the work for us, we also have to know the structure of the constructed groups.

As mentioned above, the complete locally finite $p$-group of cardinality $\aleph_{1}$ is a union of a smooth chain $\left(E_{\alpha}: \alpha<\omega_{1}\right)$ of groups. All the $E_{\alpha}$ 's are isomorphic to a particular countable group $G$. So, in fact, we just construct some self-embeddings of $G$. The main property of $G$ we use is that, for every finite subgroup $H$, the extensions $H \prec C\left(p^{n}\right)$ and $C\left(p^{n}\right) \curlywedge H$ of $H$ are also subgroups of $G$. Informally this means that finite subgroups are "nicely" embedded 
in $G$, which is used to extend homomorphisms from finite subgroups to the whole group. The self-embeddings above are just these extensions.

Originally, in [25], $G$ was the unique countable universal locally finite $p$ group. Here "universal" is similar to what is "algebraically closed" in field theory, both of which are special cases of what logicians call "existentially closed". Maier (see [18]) and Leinen (see [16, 17]) studied countable universal locally finite $p$-groups extensively, and obtained similar results as Hall did for universal locally finite groups, see [12] or [15, Chapter 6]. In particular, Maier and Leinen showed that up to isomorphism there exists a unique countable universal locally finite $p$-group, determined its automorphism group modulo locally finite automorphisms etc. Moreover, Leinen gave an explicit construction of the group. However, since we only need the property mentioned above, we are able to substitute the universal locally finite $p$-group with an iterated wreath product. This will make the construction more elementary.

The large locally finite $p$-groups we shall construct in Chapter 3 are, roughly speaking, iterated wreath products of abelian $p$-groups, which we shall call building blocks as earlier. These abelian $p$-groups form a rigid system in the sense that the only homomorphisms between them are only the unavoidable ones. Because of the wreath product construction, the large abelian subgroups are only the direct sums of conjugates of a building block, which enables us to control the embeddings together with some centralizer arguments. 


\section{Chapter 2}

\section{Complete groups under $\mathrm{CH}$}

In this chapter we consider a class $\mathcal{C}$ of locally finite $p$-groups satisfying certain closure properties and show that $\mathcal{C}$ contains a complete group of cardinality $\aleph_{1}$ (see Theorem 2.1.1). Recall that a group is complete if its centre is trivial and all of its automorphisms are inner. The construction is done in several steps. The main arguments will be discussed in Section 2.2, while the used tools will be proven later.

\subsection{Background and main results}

Thomas proved in [25] that under the diamond principle there are existentially closed complete groups of cardinality $\aleph_{1}$ in several group classes, among which we are only interested in the class of locally finite $p$-groups. We modify his proof to obtain the same result for locally finite $p$-groups under $C H$, the continuum hypothesis $2^{\aleph_{0}}=\aleph_{1}$, which is a weaker assumption. We do not need the definition of existentially closed groups. We will only use that the class of locally finite $p$-groups we are dealing with contains a countable member, is closed under union of chains and if $H$ is any finite subgroup of a group from the class then $H<C\left(p^{n}\right)$ and $C\left(p^{n}\right)$ 乙 $H$ are also.

Next we state the main theorem of this chapter and briefly discuss an immediate consequence. The rest of this chapter is devoted to the proof of the main theorem. 
For the sake of completeness (of this chapter and not the groups) and convenience of the reader, the arguments of Thomas are included. However, they are presented differently.

In what follows, $(\mathrm{CH})$ at the beginning of a statement indicates that the proof we give uses the continuum hypothesis.

Theorem 2.1.1. (CH) Let $p$ be a prime number and $\mathcal{C}$ a class of locally finite p-groups which is closed under isomorphic copies and

(a) $\mathcal{C}$ is closed under unions of chains;

(b) $\mathcal{C}$ contains a countable group;

(c) if $E \in \mathcal{C}$ and $H \subseteq E$ is a finite subgroup then $H=H e_{1} \subseteq H \curlywedge C\left(p^{n}\right) \subseteq E$ and $H \subseteq C\left(p^{n}\right)$ 乙 $H \subseteq E$ for every natural number $n$.

Then there is a complete group $G \in \mathcal{C}$ of cardinality $\aleph_{1}$.

For example, the class of locally finite $p$-groups with property (c) also satisfies the other conditions. A countable member of the class is $\bigcup_{n \in \mathbb{N}} H_{n}$ where $H_{0}:=1$ and $H_{n+1}:=C\left(p^{n}\right) \curlywedge\left(H \prec C\left(p^{n}\right)\right)$ for all $n$. Hence the following corollary is immediate:

Corollary 2.1.2. (CH) There is a complete locally finite p-group of cardinality $\aleph_{1}$.

The main improvement of Thomas' proof is that we make automorphisms to extend uniquely from certain countable subgroups (see Proposition 2.2.6 (C)).

Throughout this chapter let $\mathcal{C}$ denote a class of groups as in the assumption of Theorem 2.1.1.

\subsection{The construction}

In this section we construct a group $G$ satisfying the conclusion of Theorem 2.1.1. The group $G$ will be a union of a smooth chain of countable groups $G_{\alpha}$ from $\mathcal{C}$. The completeness of $G$ then follows from the next proposition. 
Proposition 2.2.1. Let $\left(G_{\alpha}: \alpha<\omega_{1}\right)$ be a strictly increasing smooth chain of countable groups with the following properties. Set $G=G_{\omega_{1}}:=\bigcup_{\alpha<\omega_{1}} G_{\alpha}$.

(1) If $\phi \in$ Aut $G_{\beta}$ and $G_{\alpha} \phi=G_{\alpha}$ for some $\alpha<\beta \leq \omega_{1}$ then $G_{\gamma} \phi=G_{\gamma}$ for all $\alpha \leq \gamma<\beta$.

(2) If $\phi \in \operatorname{Aut} G_{\alpha}$ and $\alpha \leq \beta \leq \omega_{1}$ then $\phi$ has at most one extension to an automorphism of $G_{\beta}$.

(3) If $\phi \in$ Aut $G_{\alpha}$ is not inner and $\alpha<\omega_{1}$ then $\phi$ does not extend to an automorphism of $G_{\beta}$ for some $\alpha<\beta<\omega_{1}$.

Then Out $G=1$.

Proof. Let $\phi \in \operatorname{Aut} G$. We prove that $\phi$ is an inner automorphism. Recall that, for any smooth increasing sequence $\left(G_{\alpha}: \alpha<\omega_{1}\right)$ of countable sets, and any bijection $\phi$ of $\bigcup_{\alpha<\omega_{1}} G_{\alpha}$, there are many $\alpha$ 's such that $G_{\alpha} \phi=G_{\alpha}$, see [5. Section 4.12]. Applying this to the present context, $\phi$ leaves $G_{\alpha}$ invariant for some $\alpha<\omega_{1}$. Moreover, it leaves all $G_{\beta}$ invariant for $\alpha<\beta<\omega_{1}$ by (1). So $\phi$ must be inner on $G_{\alpha}$ by (3). Since it extends uniquely by (2), it must be inner on $G$.

The next proposition shows how the first two conditions of the previous proposition will be guaranteed by combinatorial tools. We therefore introduce the notation

$$
S(x, H):=\{|\langle x, y\rangle|: y \in H\}
$$

where $x$ is an element and $H$ a subset of a group.

Recall that a system $\left(A_{i}: i \in I\right)$ of subsets of the set $\mathbb{N}$ of natural numbers is almost disjoint if $A_{i} \cap A_{j}$ is finite for every $i \neq j \in I$.

Proposition 2.2.2. Let $\left(A_{\alpha}: \alpha<\omega_{1}\right)$ be a collection of almost disjoint infinite subsets of $\mathbb{N}$ and $\left(G_{\alpha}: \alpha<\omega_{1}\right)$ a smooth chain of countable groups. Moreover, let $G:=\bigcup_{\alpha<\omega_{1}} G_{\alpha}$ and suppose that 
(i) for every $\alpha<\omega_{1}$

$$
\begin{aligned}
\left|S\left(x, G_{\alpha}\right)\right| & =\aleph_{0} & & \text { for all } x \in G \backslash G_{\alpha} ; \\
\left|S\left(x, G_{\alpha}\right) \backslash A_{\alpha}\right| & <\infty & & \text { for all } x \in G_{\alpha+1} \backslash G_{\alpha} ;
\end{aligned}
$$

(ii) if $\phi \in$ Aut $G_{\alpha+1}$ with $\phi \uparrow G_{\alpha}=1$ then $\phi=1$.

Then conditions (1) and (2) of Proposition 2.2.1 are satisfied.

Proof. Let the assumptions be as above, and let $\alpha<\omega_{1}$ and $x \in G \backslash G_{\alpha}$ be fixed. Then there is a $\gamma \geq \alpha$ such that $x \in G_{\gamma+1} \backslash G_{\gamma}$. We claim that, for $\alpha \leq \delta<\omega_{1}$, the set $S\left(x, G_{\alpha}\right) \backslash A_{\delta}$ is finite if and only if $\delta=\gamma$. The "if" part is just (2.3) since $S\left(x, G_{\alpha}\right) \subseteq S\left(x, G_{\gamma}\right)$. If $\delta \neq \gamma$ then

$$
\begin{aligned}
S\left(x, G_{\alpha}\right) \cap A_{\delta} & =\left(\left(S\left(x, G_{\alpha}\right) \cap A_{\delta}\right) \backslash A_{\gamma}\right) \cup\left(S\left(x, G_{\alpha}\right) \cap A_{\delta} \cap A_{\gamma}\right) \\
& \subseteq\left(S\left(x, G_{\gamma}\right) \backslash A_{\gamma}\right) \cup\left(A_{\delta} \cap A_{\gamma}\right)
\end{aligned}
$$

is finite and hence $S\left(x, G_{\alpha}\right) \backslash A_{\delta}$ is infinite.

First we show (1). Let $\phi \in \operatorname{Aut} G_{\beta}$ such that $G_{\alpha} \phi=G_{\alpha}$ for some $\alpha<$ $\beta \leq \omega_{1}$. If $x \in G_{\gamma+1} \backslash G_{\gamma}$ for some $\gamma \geq \alpha$, then $S\left(x \phi, G_{\alpha}\right) \backslash A_{\gamma}=S\left(x, G_{\alpha}\right) \backslash A_{\gamma}$ is infinite, hence $x \phi \in G_{\gamma+1} \subseteq G_{\gamma}$ by our claim. Thus we have proved that $G_{\gamma+1} \backslash G_{\gamma}$ is mapped to itself by $\phi$ for all $\alpha \leq \gamma<\beta$. It follows that $G_{\gamma} \phi=G_{\gamma}$ for all $\alpha<\gamma<\beta$ and hence (1) is proved.

Now we prove (2) indirectly: Suppose that $\phi_{1}$ and $\phi_{2}$ are different automorphisms of $G_{\beta}$ which coincide on $G_{\alpha}$ for some $\alpha<\beta \leq \omega_{1}$. Then $\phi:=\phi_{1}\left(\phi_{2}\right)^{-1}$ is identical on $G_{\alpha}$ but not on $G_{\beta}$. Since the chain $\left(G_{\gamma}: \gamma<\beta\right)$ is smooth, there is a largest $\gamma \leq \beta$ such that $\phi$ is the identity on $G_{\gamma}$. Obviously, $\gamma<\beta$. By (1), which we have already proved, $\phi \uparrow G_{\gamma+1} \neq 1$ is an automorphism of $G_{\gamma+1}$ which is identical on $G_{\gamma}$ contradicting (ii).

Adequate and admissible sets We move to consider a different element of the proof. Later this will be glued with the propositions above.

The construction of the chain $\left(G_{\alpha}: \alpha<\omega_{1}\right)$ will be done recursively. Now we attack the problem of constructing $G_{\alpha+1}$ when we have already constructed $G_{\alpha}$. 
The construction will be formulated in Proposition 2.2.6. Now we give some definitions to simplify the language.

Definition 2.2.3. Let $P<Q$ be finite p-groups and I a finite set of natural numbers. Then $I$ is adequate for $P$ and $Q$ if for every $G \in \mathcal{C}$ and every normal subgroup $1 \neq N \triangleleft G$ with $P<Q \leq G$ there is an $x \in C_{N}(P)$ such that

(i) $Q \cap Q^{x}=P$;

(ii) $|\langle a, b\rangle| \in I$ for all $a \in Q \backslash P$ and $b \in Q^{x} \backslash P$.

The existence of adequate sets will be proved in Lemma 2.3.1.

Definition 2.2.4. An infinite subset $A$ of $\mathbb{N}$ will be called admissible if it is a disjoint union

$$
A:=\bigcup_{P<Q} I_{P<Q}
$$

where the union runs through all pairs $(P, Q)$ of isomorphism classes of finite p-groups with $P<Q$, and $I_{P<Q}$ is adequate for $P$ and $Q$.

The next lemma states the existence of an almost disjoint family of admissible sets. The proof will be given in Section 2.3 .

Lemma 2.2.5. There is an almost disjoint family $\left(A_{\beta}: \beta<2^{\aleph_{0}}\right)$ of admissible subsets of $\mathbb{N}$.

Enlarging groups Now we are ready to formulate the main step of the construction: constructing $G_{\alpha+1}$ from $G_{\alpha}$.

Proposition 2.2.6. Let $G \in \mathcal{C}$ be a countable group and $A$ an admissible set. Then there is a group $G^{1} \supsetneq G$ such that $G^{1}$ is isomorphic to $G$ and for all $x \in G^{1} \backslash G$ :

(A) $\{y \in G||\langle x, y\rangle \mid=n\}$ is a finite set for all natural numbers $n$;

(B) $S(x, G) \backslash A$ is finite; 
(C) if $\phi \in$ Aut $G^{1}$ and $\phi \uparrow G=1$ then $\phi=1$.

Moreover, if $f \in$ Aut $G$ is not an inner automorphism then $G^{1}$ can be chosen so that $f$ does not extend to an automorphism of $G^{1}$.

In the proof, we will use the following lemma, which characterizes outer automorphisms of $G$. The proof will be included in Section 2.4.

Lemma 2.2.7. (25] Theorem 2) Let $E$ be a group of the class $\mathcal{C}$ and $P$ a finite subgroup of $E$. Then every automorphism of $E$ which leaves every finite subgroup containing $P$ invariant is inner.

Next we present an easy observation about normal subgroups.

Lemma 2.2.8. If $G \in \mathcal{C}$ then every non-trivial normal subgroup of $G$ is infinite.

Proof. If $H$ is a finite subgroup of $G$ then, by condition (c) of Theorem 2.1.1, the extension $H$ ح $C(p)$ of $H$ is also contained in $G$. Since $H$ is not normal in $H$ 乙 $C(p)$, it cannot be normal in $G$ either.

We can now prove the proposition.

Proof of Proposition 2.2.6. We suppose that $A$ is given in the form of 2.4.

Since $G^{1}$ should be isomorphic to $G$, constructing $G^{1}$ actually means constructing a self-embedding of $G$. For suppose we have a $\vartheta: G \rightarrow G$ self-embedding of $G$, such that $G$ is an extension of $G \vartheta$ satisfying all the requirements of the proposition replacing $G^{1}$ by $G$, the group $G$ by $G \vartheta$ and the automorphism $f$, if given, by the automorphism $g$ of $G \vartheta$ determined by $\vartheta g=f \vartheta$. Then we can choose $G^{1}$ to be a group isomorphic to $G$ via a map $\rho: G \cong G^{1}$ such that $\vartheta \rho$ is an inclusion. Then $G^{1}$ will satisfy all the requirements of the proposition.

First we choose a decreasing sequence $\left\{N_{n}\right\}_{n \in \omega}$ of infinite normal subgroups of $G$ such that $\bigcap_{n \in \omega} N_{n}=1$. To do so, recall from [15] that $G$, as every group, has a chief series, i.e. a system $\left(M_{i}, K_{i}\right)_{i \in I}$ of normal subgroups where $I$ is an ordered set such that the following holds. For all $i, j \in I$ we 
have $K_{i} \subseteq M_{j}$ if $i<j$. Moreover, for all $i \in I M_{i} \subseteq K_{i}$ but no normal subgroup lies strictly between $M_{i}$ and $K_{i}$. Lastly, $G \backslash\{1\}=\bigcup_{i \in I} K_{i} \backslash M_{i}$. Also recall that the chief factors $K_{i} / M_{i}$ of any locally finite $p$-group are cyclic of order $p$ (see [15, Corollary 1.B.8]) and that all non-trivial normal subgroups of $G$ are infinite (Lemma 2.2.8). Thus all the $M_{i}$ 's are infinite $(i \in I)$.

Now one can choose the $N_{n}$ 's among the $M_{i}$ 's recursively: enumerate the non-trivial elements of $G$, say $G \backslash\{1\}=\left\{a_{n}: n \in \omega\right\}$ and choose $N_{n}$ to be an $M_{i}$ such that $a_{n} \notin N_{n}$ and, if $n>0$, then $N_{n-1} \supseteq N_{n}$. For example, if $N_{n-1}=M_{i}$ and $j$ is the unique element of $I$ such that $a_{n} \in K_{j} \backslash M_{j}$ then one can set $N_{n}:=M_{\min \{i, j\}}$. This ensures that $\bigcap_{n \in \omega} N_{n}$ is trivial since it does not contain any of the $a_{n}$ 's.

The embedding $\vartheta$ will be constructed with the help of two increasing chains of finite subgroups $H_{n}$ and $E_{n}=H_{n}^{x_{n}}$ where $\left\langle H_{n}, x_{n}\right\rangle \leq H_{n+1}, x_{n} \in$ $C_{N_{n}}\left(E_{n}\right)$ and $E_{n+1} \cap H_{n+1}=E_{n}$.

We construct the $E_{n}$ 's, $H_{n}$ 's and $x_{n}$ 's recursively. Let us fix an enumeration of the elements of $G$, say, $G=\left\{g_{n} \mid n=1,2, \ldots\right\}$. Let $E_{0}=H_{0}:=1$ (or any other finite subgroup) and $x_{0}:=1$. Suppose $E_{n-1}, H_{n-1}$ and $x_{n-1}$ are defined. Let $H_{n}$ be such that it contains $E_{n-1}, H_{n-1}, x_{n-1}$ and $g_{n}$. Such a finite $H_{n}$ exists because $G$ is locally finite. Moreover, if $f$ is given, let us choose $H_{n}$ such that it is not invariant under $f$. This is possible by Lemma 2.2.7. Since $I_{E_{n-1}<H_{n}}$ is adequate for $E_{n-1}$ and $H_{n}$, we can choose $x_{n} \in C_{N_{n}}\left(E_{n-1}\right)$ such that $E_{n}:=H_{n}^{x_{n}}$ and $H_{n}$ satisfy (ii) and (ii) of Definition 2.2.3 with $I:=I_{E_{n-1}<H_{n}}, P:=E_{n-1}, Q:=H_{n}, x:=x_{n}$ and $Q^{x}=E_{n}$.

Since $g_{n} \in H_{n}$, the union of $H_{n}$ 's is the whole group $G$. Define the selfembedding $\vartheta$ of $G$ by letting it be conjugation by $x_{0} x_{1} x_{2} \ldots x_{k}$ on $H_{n}$ for any $k \geq n$. Note that $x_{0}, \ldots, x_{n-1}$ are elements of $H_{n}$ so $H_{n}^{x_{0} \ldots x_{n}}=H_{n}^{x_{n}}=E_{n}$ and $E_{n}$ is centralized by $x_{i}$ for $i>n$. This shows that $\vartheta$ is well defined and $H_{n} \vartheta=E_{n}$.

We are going to check $(\mathrm{A})$ and $(\mathrm{B})$ replacing $G^{1}$ by $G$ and $G$ by $G \vartheta$. Therefore let $x \in G \backslash G \vartheta$. There is a smallest natural number $n$ such that $x \in H_{n}$. For $k \geq n$ we have $x \in H_{k} \backslash E_{k-1}$, so by the construction $S\left(x, E_{k} \backslash\right.$ 
$\left.E_{k-1}\right) \subseteq I_{E_{k-1}<H_{k}}$. This shows that in the union

$$
S(x, G \vartheta)=S\left(x, E_{n-1}\right) \cup \bigcup_{k \geq n} S\left(x, E_{k} \backslash E_{k-1}\right)
$$

the big union is disjoint and is contained in $A$. Hence $\mathrm{A}$ is immediate. We also get $S(x, G \vartheta) \backslash A \subseteq S\left(x, E_{n-1}\right)$ is finite, so $(\mathrm{B})$ holds also.

Finally, we prove that if $\phi \in \operatorname{Aut} G$ and $(G \vartheta) \phi=G \vartheta$ then $E_{n} \phi=E_{n}$ for all but finitely many $n$. Moreover, if $\phi$ is identical on $G \vartheta$ then $\phi=1$. This will prove property (C).

It will also follow that, if $f$ is given, the automorphism $g$ of $G \vartheta$ determined by $\vartheta g=f \vartheta$ does not extend to an automorphism of $G$. The reason is that, for all natural number $n, H_{n} f \neq H_{n}$ by construction, and so $E_{n} g=H_{n} \vartheta g=$ $H_{n} f \vartheta \neq H_{n} \vartheta=E_{n}$.

Now let $\phi \in$ Aut $G$ be given with $(G \vartheta) \phi=G \vartheta$. Choose an arbitrary $x \in G \backslash G \vartheta$. Then $x, x \phi \in H_{m}$ for $m$ large enough. The sets $I_{E_{n-1}<H_{n}}(n \in \mathbb{N})$ are pairwise disjoint. So there is an $N$ such that, for all natural numbers $n>N$, the set $I_{E_{n-1}<H_{n}}$ is disjoint from $S\left(x, E_{m-1}\right)$ and $S\left(x \phi, E_{m-1}\right)$. Since $S\left(x, E_{k} \backslash E_{k-1}\right), S\left(x \phi, E_{k} \backslash E_{k-1}\right) \subseteq I_{E_{k-1}<H_{k}}$ for all $k \geq m$, it follows that the sets $S\left(x, E_{N}\right) \cup S\left(x \phi, E_{N}\right)$ and $I_{E_{n-1}<H_{n}}$ are pairwise disjoint for $n>N$.

Let $y \in G \vartheta$. If $y \in E_{N}$ then $|\langle x \phi, y \phi\rangle|=|\langle x, y\rangle| \in S\left(x, E_{N}\right)$ and hence $y \phi \in E_{N}$. If $y \in E_{n} \backslash E_{n-1}$ for some $n>N$ then $|\langle x \phi, y \phi\rangle|=|\langle x, y\rangle| \in$ $I_{E_{n-1}<H_{n}}$ and hence $y \phi \in E_{n} \backslash E_{n-1}$. Thus we have proved that $E_{N} \phi \subseteq E_{N}$ and $\left(E_{n} \backslash E_{n-1}\right) \phi \subseteq E_{n} \backslash E_{n-1}$ for all $n>N$. It follows that $E_{n} \phi=E_{n}$ for all $n \geq N$.

To finish the proof, we consider $\phi \in \operatorname{Aut} G$ with $\phi \uparrow G \vartheta=1$. We have to prove that $\phi=1$.

To do so, note first that every normal subgroup is invariant under $\phi$. In fact, even every conjugacy class is invariant under $\phi$, since $\phi$ is the identity on $G \vartheta$ and every conjugacy class intersects $G \vartheta$. To see the last claim, note that if $x \in G$ then $x \in H_{n}$ for some $n>0$ and hence $x^{x_{n}} \in E_{n} \subseteq G \vartheta$. This shows that the conjugacy class of $x$ intersects $G \vartheta$.

Now we prove that $\phi=1$. Therefore let $x \in G$ be arbitrary. We show that $x \phi=x$. There is an $N$ such that such that $x \in H_{N}$. Let $n \geq N$ be an 
arbitrary natural number. Then $x \in H_{n}$ and so $x^{x_{n}} \in E_{n} \subseteq G \vartheta$. Since $\phi$ is identical on $G \vartheta$ by hypothesis, we have

$$
x^{x_{n}}=\left(x^{x_{n}}\right) \phi=(x \phi)^{x_{n} \phi} .
$$

Expressing $x^{-1} \cdot x \phi$ using this equation, we get

$$
x^{-1} \cdot x \phi=x^{-1} \cdot x^{x_{n} \cdot\left(x_{n} \phi\right)^{-1}} \in N_{n} .
$$

Here we used that $\phi$ preserves normal subgroups and so, in particular, $x_{n} \phi \in$ $N_{n}$ since $x_{n} \in N$.

So we have proved that $x^{-1} \cdot x \phi \in \bigcap_{n \geq N} N_{n}=1$. In other words, $x \phi=x$ and this finishes the proof.

Proof of the main theorem Now we have done all preliminary work to prove the main theorem of this chapter.

Proof of Theorem 2.1.1. First, we choose a sequence of almost disjoint admissible sets $\left(A_{\alpha}: \alpha<\omega_{1}\right)$, which exists by Lemma 2.2 .5 .

Moreover, we fix a bijection $\chi: \omega_{1} \times \omega_{1} \rightarrow \omega_{1}$ such that $(\alpha, \beta) \chi \geq \alpha$ for all $\alpha, \beta<\omega_{1}$.

We shall construct a sequence $\left(G_{\alpha}: \alpha<\omega_{1}\right)$ of countable groups from the given class $\mathcal{C}$ recursively.

Let $G_{0}$ be any countable member of $\mathcal{C}$. If $\alpha$ is a limit ordinal put $G_{\alpha}:=$ $\bigcup_{\beta<\alpha} G_{\beta}$. Since $\mathcal{C}$ is closed under unions of chains, $G_{\alpha} \in \mathcal{C}$. Since the union of countable many countable groups is countable, $G_{\alpha}$ must be countable.

Let us construct $G_{\alpha+1}$ when the groups $\left\{G_{\beta}: \beta \leq \alpha\right\}$ are already given. Let $\left(f_{(\alpha, \beta)}: \beta<\gamma_{\alpha}\right)$ be a fixed enumeration of the non-inner automorphisms of $G_{\alpha}$ for some $\gamma_{\alpha} \leq 2^{\aleph_{0}}=\aleph_{1}$. We apply Proposition 2.2.6 with $G:=G_{\alpha}$, $A:=A_{\alpha}$. If $f_{\alpha \chi^{-1}}$ exists and has an extension to an outer automorphism of $G_{\alpha}$, we also let $f$ to be such an extension. We set $G_{\alpha+1}:=G_{\alpha}^{1}$. Note that $G_{\alpha+1}$ is isomorphic to $G_{\alpha}$ and hence is contained in $\mathcal{C}$.

We claim that $G:=\bigcup_{\alpha<\omega_{1}} G_{\alpha}$ is a complete group of cardinality $\aleph_{1}$ from the class $\mathcal{C}$. It is obvious that $G$ has cardinality $\aleph_{1}$. Since $\mathcal{C}$ is closed under 
union of chains, $G \in \mathcal{C}$. Since the elements of $\mathcal{C}$ are locally finite and satisfy condition (C) of Theorem 2.1.1, all of them have trivial centre. (If $x$ is a non-zero element of $E \in \mathcal{C}$ then $x$ does not centralize $\langle x\rangle \prec C(p) \subseteq E$.) So we only have to prove that all automorphisms of $G$ are inner.

As a first step, we show that the so constructed chain $\left(G_{\alpha}: \alpha<\omega_{1}\right)$ satisfies the conditions of Proposition 2.2.2. Condition (ii) is satisfied by the construction and Proposition 2.2.6 (C). Condition (2.3) follows from (B).

Let $x \in G \backslash G_{\alpha}$ for some $\alpha<\omega_{1}$. Then $x \in G_{\beta+1} \backslash G_{\beta}$ for some $\alpha \leq \beta<\omega_{1}$. By A and since $G_{\alpha} \subseteq G_{\beta}$ is infinite, $S\left(x, G_{\alpha}\right)$ must be an infinite set. Thus 2.2 is also satisfied.

So we can apply Proposition 2.2.2 to obtain that (1) and (2) of Proposition 2.2.1 hold. We are going to show that (3) also holds and thus Out $G=1$ by the proposition, which will finish the proof.

Let $\phi$ be a non-inner automorphism of $G_{\alpha}$ for some $\alpha<\omega_{1}$. Then $\phi=$ $f_{(\alpha, \beta)}$ for some $\beta<\omega_{1}$. Let $\gamma:=\chi(\alpha, \beta)$. Suppose that $\phi$ extends to an automorphism of $G_{\gamma}$. Then there is an extension $f$ such that $f$ does not extend to an automorphism of $G_{\gamma+1}$, because $G_{\gamma+1}$ was so constructed. We claim that $\phi$ does not extend to an automorphism of $G_{\gamma+1}$. Otherwise let $\psi$ be such an extension. Since $G_{\alpha} \psi=G_{\alpha}$, we must have $G_{\gamma} \psi=G_{\gamma}$ by (1). By (2), the extension of $\phi$ to $G_{\gamma}$ is unique and hence $f=\psi \uparrow G_{\gamma}$. Hence $\psi$ is an extension of $f$, a contradiction.

Although the main theorem is proved, we still owe the proofs of two lemmas used. This will be done in the remaining two sections.

\subsection{The choice of almost disjoint sets $A_{\beta}$}

We are going to prove Lemma 2.2.5, i.e. that an almost disjoint family of admissible sets of cardinality $2^{\aleph_{0}}$ exists. The proof is a combination of combinatorial and group theoretic techniques. First we formulate and prove the group theoretic part as a separate lemma and then present the combinatorial methods. 
Lemma 2.3.1. Let $P<Q$ be finite $p$-groups and $n$ a natural number. Then there is a group $H>Q$ and $x \in C_{H}(P)$ such that:

(1) $Q \cap Q^{x}=P$;

(2) if $a \in Q \backslash P$ and $b \in Q^{x} \backslash P$ then $|\langle a, b\rangle|>n$;

(3) if $G \in \mathcal{C}, 1 \neq N \triangleleft G$ and $Q<E$ then the natural inclusion of $Q$ into $E$ extends to an embedding of $H$ into $E$ such that the image of $x$ lies in $N$.

In particular, the following set is adequate for $P$ and $Q$ :

$$
I_{P<Q}:=\left\{|\langle a, b\rangle|: a \in Q \backslash P, b \in Q^{x} \backslash P\right\}
$$

Proof. It clearly follows from the rest of the lemma that $I_{P<Q}$ is adequate for $P$ and $Q$. So we only have to prove the other parts of the lemma.

We will choose $H:=C\left(p^{N}\right) \curlywedge Q$ for $N$ large enough such that $p^{N}>|Q|$, $n$. Then this group satisfies the requirements of the lemma.

We are going to make computations in the wreath product $C\left(p^{N}\right) \curlywedge Q=$ $\bigoplus_{a \in Q} C\left(p^{N}\right) e_{a} \rtimes Q$, so this is a good point to introduce our notation. We let $g$ denote a generator of the cyclic group $C\left(p^{N}\right) e_{1}$. The group $Q$ acts on $B:=\bigoplus_{a \in Q} C\left(p^{N}\right) e_{a}$ by conjugation, i.e. by permuting the coordinates by the right regular representation. Therefore $B$ is a $\mathbb{Z} Q$-module, where $\mathbb{Z} Q$ is the group ring of $Q$ over the integers. If $y \in B$ and $r \in \mathbb{Z} Q$ then the action of $r$ on $y$ is denoted by $y^{r}$. We denote the sum of elements of $P$ in $\mathbb{Z} Q$ again by $P$ as an abuse of language.

Now we choose the $x$ for the lemma:

$$
x:=g^{P} .
$$

If $q \in Q$ then

$$
q^{x}=x^{1-q} q=x^{P(1-q)} q
$$

So $q^{x} \in Q$ if and only if $q \in P$, and in this case $q^{x}=q$. Hence $x$ centralizes $P$ and $Q \cap Q^{x}=P$. 
We turn to prove (2). Choose $a, b$ as in (2). We claim that $|\langle a, b\rangle| \geq$ $p^{N}>n$. First of all, we can suppose that $G:=\langle a, b\rangle$ normalizes $P \cap G$. To see this, note that $N_{Q \cap G}(P \cap G) \gtrless P \cap G$ and $N_{Q^{x} \cap G}(P \cap G) \gtrless P \cap G$ since $Q \cap G$ and $Q^{x} \cap G$ are finite $p$-groups and hence nilpotent. So we can select $a^{\prime} \in N_{Q \cap G}(P \cap G) \backslash(P \cap G)$ and $b^{\prime} \in N_{Q^{x} \cap G}(P \cap G) \backslash(P \cap G)$. Then $G^{\prime}:=\left\langle a^{\prime}, b^{\prime}\right\rangle \subseteq G$ normalizes $P \cap G$ and $P \cap G^{\prime}=(P \cap G) \cap G^{\prime}$.

Second, we can choose a non-trivial word $a^{i_{1}} c^{j_{1}} \ldots a^{i_{l}} c^{j_{l}}$ in $P$, with $i_{k}$, $j_{k}>0$ for all $1 \leq k \leq l$, such that no proper subword of it is in $P$. (Here again $b=c^{x}$.) Therefore we consider words of $a$ and $c$ which are in $P$ and do not contain a subword consisting of only $a$ 's or $c$ 's which is also in $P$. Such a word exists, for example $(a c)^{k}$ where $k$ is the order of $a c$. So there is a word $w$ among these with minimal length. We claim that there is a word among the ones with minimal length which starts with $a$ and ends with $c$. For if $w$ starts with $c$ then we can write it as $w=c^{i} x$ where $x$ is a word starting with $a$. Since $c$ normalizes $P$, we have $x c^{i} \in P$ and this word now starts with $a$ and ends with $c$. Note that it cannot happen that $x c^{i}=y c^{j}$ for a word $y$ and $c^{j} \in P$ because then $y$ would be a smaller word contained in $P$. We can similarly handle the case when $w$ ends with $a$.

So we may suppose that $w$ starts with $a$ and ends with $c$ and thus has the form $w=a^{i_{1}} c^{j_{1}} \ldots a^{i_{l}} c^{j_{l}}$. No proper subword of $w$ is in $P$ by the minimal length of $w$.

We claim that the element

$$
y:=a^{i_{1}} b^{j_{1}} \ldots a^{i_{l}} b^{j_{l}} \in G
$$

has order at least $p^{N}$, which will finish the proof. First we compute $y$ :

$$
\begin{aligned}
y & =a^{i_{1}} c^{j_{1}} \ldots a^{i_{l}} c^{j_{l}} x^{(1-c) \sum_{k=1}^{l}\left(c^{j_{k}-1}+\cdots+1\right) \prod_{t>k} a^{i_{t}} c^{j_{t}}} \\
& =w \cdot \underbrace{g^{P \sum_{k=1}^{l}\left(1-c^{j_{k}}\right) \prod_{t>k} a^{i_{t}} c^{j_{t}}}}_{z} .
\end{aligned}
$$

where $w=a^{i_{1}} c^{j_{1}} \ldots a^{i_{l}} c^{j_{l}} \in P$. Here $z \in \bigoplus_{q \in Q} C\left(p^{N}\right)$ coincides with $g^{P}=x$ on the coordinates belonging to $P$. Hence $z^{k}$ coincides with $x^{k}$ on $P$ using that $w \in P$. Thus $z$ must have order at least $p^{N}$. 
Finally, let us prove (3). Since $N$ is infinite by Lemma 2.2.8, there exists an $y \in N \backslash Q$. By (c) of Theorem 2.1.1, the wreath product $C\left(p^{N}\right) \imath\langle y, Q\rangle=$ $\bigoplus_{q \in\langle y, Q\rangle} C\left(P^{N}\right) e_{q} \rtimes\langle y, Q\rangle$ is contained in $G$. We define an embedding of $H=C\left(p^{N}\right) \imath Q=\bigoplus_{q \in Q} C\left(p^{N}\right) e_{q} \rtimes Q$ into this group by letting it be identical on $Q$ and for all $q \in Q$ mapping $g e_{q}$ into $g e_{q}^{-1}+g e_{y q}=\left[g e_{q}, y^{q}\right](\in N)$. This embedding is well-defined and $\bigoplus_{q \in Q} c\left(p^{N}\right) e_{q}$ is mapped into $N$. Especially, $x$ is mapped into $N$ by 2.9 .

Having constructed many adequate sets in the previous proof, we are able to build from these an almost disjoint family of admissible sets in the next proof.

Proof of Lemma 2.2.5. Recall that an admissible set is a disjoint union of adequate sets. So, it is natural to first construct a lot of adequate sets and then the admissible ones.

Let $\left(P_{n}, Q_{n}\right)(n \in \omega)$ be an enumeration of all the pairs $(P, Q)$ of isomorphism classes of finite $p$-groups with $P<Q$. There are countably infinite many such pairs.

For every $m \in \omega$ and any function $f:\{0,1, \ldots, m-1\} \rightarrow\{0,1\}$ we will select a set $I_{P_{m}<Q_{m}}^{f}$ adequate for $P_{m}$ and $Q_{m}$, such that these sets are pairwise disjoint for all $f$ and $m$. Altogether, we have to choose countably many sets, so we enumerate them in a sequence of type $\omega$ and then select the sets recursively. Hence, when we select $I_{P_{m}<Q_{m}}^{f}$, we have to make sure that this is adequate for $P_{m}$ and $Q_{m}$ and is disjoint from the finitely many previously selected finite sets. We can ensure the disjointness by choosing all the elements of $I_{P_{m}<Q_{m}}^{f}$ greater than some $n \in \omega$. Apply Lemma 2.3.1 to $P:=P_{m}, Q:=Q_{m}$ and $n$. Then we can set $I_{P_{m}<Q_{m}}^{f}:=I_{P<Q}$ using the notations of the lemma.

For every function $g: \omega \rightarrow\{0,1\}$ put

$$
A_{g}:=\bigcup_{n \in \omega} I_{P_{n}<Q_{n}}^{g \nmid n}
$$

Then $A_{g}$ is clearly admissible. Moreover, the $A_{g}$ 's form an almost disjoint family of size $2^{\aleph_{0}}$ when $g$ runs through all functions $\omega \rightarrow\{0,1\}$. For if $g_{1} \neq g_{2}$ 
then let $k$ be the smallest natural number such that $k g_{1} \neq k g_{2}$. Then

$$
A_{g_{1}} \cap A_{g_{2}}=\bigcup_{n \leq k} I_{P_{n}<Q_{n}}^{g_{1} \mid n}
$$

is finite. This finishes the proof.

\subsection{Characterization of outer automorphisms}

In this section we prove Lemma 2.2.7. Our arguments are based on the structure of some special automorphisms of wreath products. We collect the necessary facts together in the following lemma, which is partly extracted from [25]. But we give a modified proof, which avoids the deep characterization of automorphisms of wreath products established by Houghton [14].

Lemma 2.4.1. Let $B$ and $C$ be any groups and $B \imath C=\bigoplus_{c \in C} B e_{c} \rtimes C$ their restricted wreath product. Let $\phi$ be an automorphism of $B$ ? $C$. Then

(i) If $\phi$ leaves every subgroup containing $B e_{1}$ invariant, then it also leaves invariant every subgroup of $B e_{c}$ for every $1 \neq c \in C$.

(ii) If $\phi$ is identical on $B e_{c}$ for some $c \in C$ and $B e_{1} \phi=B e_{1}$, then it is inner on $B e_{1}$.

(iii) If $\phi$ leaves every subgroup of $B<C$ invariant and both $B$ and $C$ are non-trivial, then $\phi$ is the identity.

Proof. First we prove (ii). Note that the conjugates of $B e_{1}$ are the groups $B e_{c}$ for $c \in C$. Since $B e_{1}$ is invariant, these groups are permuted by $\phi$. Let $H \subseteq B e_{c}$ be a subgroup for some $1 \neq c \in C$. Then $H \phi \subseteq B e_{d}$ for some $d \neq 1$. By hypothesis, $B e_{1} \oplus H \phi=\left(B e_{1} \oplus H\right) \phi=B e_{1} \oplus H$. This implies $H \phi=H$.

Next we prove (ii). Let $x \in B e_{1}$. Then $x^{c} \in B e_{c}$ and hence $x^{c}=\left(x^{c}\right) \phi=$ $(x \phi)^{c \phi}$. Thus $x \phi=x^{c(c \phi)^{-1}}$ for all $x \in B e_{1}$. Since conjugation by every element of $B$ < $C$ induces an inner automorphism on $B e_{1}$, provided $B e_{1}$ is invariant, $\phi$ is inner on $B e_{1}$. 
Finally, we prove (iii). By hypothesis, $\phi$ maps every element to some of its powers. So for all $b \in B \backslash\{1\}$ and $c \in C \backslash\{1\}$

$$
\begin{aligned}
\left(b e_{1} c\right) \phi & =\left(b e_{1} c\right)^{n} \\
\left(b e_{1}\right) \phi & =b^{k} e_{1} \neq 1 \\
c \phi & =c^{m}
\end{aligned}
$$

for some non-zero integers $n, m$ and $k$. Since $\left(b e_{1} c\right) \phi=\left(b e_{1}\right) \phi \cdot c \phi$ we get

$$
b^{k} e_{1} c^{m}=\left(b e_{1} c\right)^{n}=b\left(e_{1}+e_{c}+\ldots+e_{c^{n-1}}\right) c^{n} .
$$

Let $0=i_{1}<\ldots<i_{l} \leq n-1$ be the integers $i$ between 0 and $n-1$ for which $c^{i}=1$. Then the $e_{1}$-coordinate of the right-hand side of (2.17), which is $b^{l}$, equals the $e_{1}$-coordinate, $b^{k}$, of the left-hand side, i.e. $b^{l}=b^{k}$. The integers $i$ between 0 and $n-1$ for which $c^{i}=c$ are $i_{1}+1<\ldots<i_{l-1}+1$ and also $i_{l}+1$ if $i_{l}<n-1$. Hence the $e_{c}$ 'th coordinate of the right-hand side of (2.17), which must be 1 , is $b^{l-1}=1$ if $i_{l}=n-1$ and $b^{l}=1$ if $i_{l}<n-1$. But since $b^{l}=b^{k} \neq 1$, this implies that $i_{l}=n-1$ and $b^{l-1}=1$, and hence $b^{l}=b^{k}=b$, $c^{n-1}=1$ and $c^{m}=c^{n}=c$. So we have proved that $\left(b e_{1}\right) \phi=b e_{1}$ and $c \phi=c$ for all non-trivial $b \in B$ and $c \in C$. Since these elements generate $B$ ? $C$, we must have $\phi=1$.

Knowing enough about the automorphisms of wreath products, we can easily prove Lemma 2.2.7 now.

Proof of Lemma 2.2.7. Let $E \in \mathcal{C}, P$ a finite subgroup and $\phi$ an automorphism of $E$. Assume that $\phi$ leaves every finite subgroup containing $P$ invariant. We will prove that $\phi$ is conjugation by an element of $P$.

Suppose the contrary for contradiction. Since $P$ is finite, there is a finite subset of $E$ such that $\phi$ differs already on this set from all the conjugations by an element of $P$. Since $E$ is locally finite, there is a finite subgroup $A$ containing $P$ and this finite set. We shall show that $\phi$ is conjugation by an element of $P$ on $A$, which leads to a contradiction.

Apply Lemma 2.4.1 to $(C(p)$ 乙 $A) \prec C(p)$ and $\phi$. Then $\phi$ leaves invariant 
every subgroup of $(C(p) \prec A) e_{c}(c \neq 1)$ by (i), hence is identical on these groups by (iii). Hence $\phi$ is inner on $(C(p) \prec A) e_{1}=C(p) \prec A$ by (iii).

Now consider $\phi$ on $C(p) 2 A$. It is inner on $A$, and the conjugation is by an element of $P$ since $\langle g, P\rangle$ is invariant where $g$ is a generator of $C(p) e_{1}$.

Now the proof of the main theorem of this chapter is complete. 


\section{Chapter 3}

\section{Outer embeddings of large locally finite $p$-groups}

In this chapter we are going to construct a fully rigid system of locally finite $p$-groups. With the help of this construction, we characterize the outer embedding monoids of locally finite $p$-groups with trivial centre.

In Section 3.1 we state the main results and we prove them step by step in the following sections. In particular, Section 3.2 consist of an abstract monoid construction, which enables us to characterize the monoids which appear as an outer embedding monoid of some group as those in which every left or right invertible element is invertible. In Section 3.3 we construct a fully rigid system of abelian $p$-groups, from which we build our locally finite $p$-groups in Section 3.4 .

\subsection{Preliminaries and the main theorem}

Let $\operatorname{Emb}(A, B)$ denote the set of embeddings of the group $A$ into the group $B$.

Recall that the outer automorphism group Out $G$ of a group $G$ is the factor of the automorphism group Aut $G$ by the normal subgroup Inn $G$ of 
inner automorphisms. It is a factor by the congruence $\sim$ :

$$
a \sim b: \Longleftrightarrow \exists g^{*} \in \operatorname{Inn} G: a=b g^{*} .
$$

As we mentioned in the introduction, this formula also gives a congruence on the monoid Emb $G$ of self-embeddings of $G$. We call the factor OutEmb $G:=$ $\operatorname{Emb} G / \sim$ the outer embedding monoid of $G$.

A question arises naturally: Which monoids can appear as an outer embedding monoid of a group? Obviously, in such monoids every left or right invertible element has to be invertible. In fact, we will show in Corollary 3.1.4 that every monoid with this property appears as an outer embedding monoid of some group.

We now introduce a factor of a monoid by a subgroup, which will be useful in formulating our results:

Definition 3.1.1. Let $M$ be a monoid and $B$ a subgroup of $M$. Assume that $M$ acts on $B$ by group homomorphisms such that

$$
b \cdot m=m \cdot b^{m},
$$

where $b^{m}$ is the action of $m$ on $b$ and $\cdot$ is the monoid operation.

Then $\sim$ is a congruence of $M$ defined by

$$
m_{1} \sim m_{2}: \Longleftrightarrow \exists b \in B m_{1} b=m_{2}
$$

We define the factor $M / B$ by

$$
M / B:=M / \sim
$$

For example, if $M$ is a group then $B$ is automatically a normal subgroup and $M$ must act on it by conjugation due to $(3.2)$. Then $M / B$ is the usual factor of $M$ by $B$.

For another example, let $G$ be a centreless group. For $g \in G$ let $g^{*}$ denote the conjugation by $g$. In other words, for every $h \in G$, let $h g^{*}=g^{-1} h g$. Set $M=\operatorname{Emb} G$, the monoid of self-embeddings of $G$. A natural action of $M$ 
on $B:=\operatorname{Inn} G$ is given by $\left(g^{*}\right)^{m}:=((g) m)^{*}$. Then $M / B=$ OutEmb $G$, the group of outer embeddings of $G$ as defined above.

After these preliminaries, we formulate the main results of this chapter.

Theorem 3.1.2. Let $\lambda$ and $\kappa$ be cardinals such that $\lambda=\lambda^{\aleph_{0}}<\kappa$ and $\aleph_{0}<\operatorname{cf} \kappa \leq 2^{\lambda}$. Moreover, let $M$ be a monoid of cardinality less than $\kappa$ in which right cancellation holds. Assume that $B$ is a subgroup of $M$ and $M$ is acting on $B$ such that for all $b \in B$ and $m \in M$

$$
b \cdot m=m \cdot b^{m}
$$

where $b^{m}$ denotes the action of $m$ on $b$.

Then there is a system $P_{X}(X \subseteq \kappa)$ of locally finite p-groups of cardinality $\kappa$ such that, for some wreath product $P_{\kappa} \rtimes B$, the subgroups $G_{X}:=P_{X} \cdot B$ $(X \subseteq \kappa)$ satisfy:

(1) $P_{X} \subseteq P_{Y}$ if and only if $X \subseteq Y$;

(2) $\operatorname{Emb}\left(G_{X}, G_{Y}\right)= \begin{cases}M \cdot \operatorname{Inn} G_{Y} & \text { if } X \subseteq Y, \\ \emptyset & \text { if } X \nsubseteq Y ;\end{cases}$

(3) $M \cap \operatorname{Inn} G_{\kappa}=B$.

In particular, OutEmb $G_{X} \cong M / B$ for all $X \subseteq \kappa$.

There are two immediate consequences:

Corollary 3.1.3. For every monoid $H$, there is a locally finite p-group with trivial centre and outer embedding monoid $H$, if and only if there is a monoid $M$ with right cancellation, a locally finite $p$-subgroup $B$ of $M$ and an action of $M$ on $B$ such that $b \cdot m=m \cdot b^{m}$, for all $b \in B$ and $m \in M$, and $H \cong M / B$.

Note that if $G$ is a group with trivial centre then we can write OutEmb $G$ as $\operatorname{Emb} G / \operatorname{Inn} G$ where the action of $\operatorname{Emb} G$ on $\operatorname{Inn} G \cong G$ is the natural one. Hence the condition of the corollary is necessary. It is also sufficient by the theorem above.

For arbitrary groups we can give a satisfactory characterization of outer embedding monoids: 
Corollary 3.1.4. A monoid $H$ appears as an outer embedding monoid of some group if and only if every left or right invertible element of $H$ is invertible.

The last corollary will be proved in the next section. The remaining sections are devoted to the proof of the main theorem.

\subsection{Construction of monoids}

Theorem 3.1 .2 and the next proposition imply Corollary 3.1.4. Our aim in this section is to prove this representation proposition.

Proposition 3.2.1. Let $H$ be a monoid in which every left or right invertible element is invertible.

Then there is a monoid $M$ with right cancellation, and a subgroup $B$ of $M$ and an action of $M$ on $B$ such that

$$
\begin{gathered}
b \cdot m=m \cdot b^{m} \quad \text { for all } b \in B \text { and } m \in M \\
M / B \cong H .
\end{gathered}
$$

Moreover, $M$ can be chosen to have cardinality $|M| \leq|H| \cdot \aleph_{0}=: \lambda$.

Proof. Let $G$ be the group of invertible elements of $H$. Note that, by hypothesis, $H \backslash G$, the set difference of $H$ and $G$, is a semigroup on which $G$ acts by conjugation. Let $F$ be the free semigroup generated by $H \backslash G$ and set

$$
N:=F \rtimes G
$$

where the action of $G$ on $F$ is permuting the generators by conjugation. Note that there is a natural epimorphism $\varphi: N \rightarrow H$ which is the identity on $G$ and $H \backslash G$.

Let us define a surjective map by

$$
\begin{aligned}
\pi: S:=\lambda \times H \times N & \rightarrow H, \\
(\alpha, h, x) & \mapsto h \cdot(x \varphi) .
\end{aligned}
$$


Now $M$ will be a part of the monoid of injective mappings from $S$ to itself preserving $\operatorname{ker} \pi$, so $M$ will have right cancellation. These mappings induce a self-embedding of the set $H$ into itself via $\pi$. We identify $N$ with a submonoid of the monoid of self-embeddings of $S$ via identifying $n \in N$ with $n^{R}$, which is defined by $(\alpha, h, x) n^{R}:=(\alpha, h, x n)$. Then $n^{R}$ induces on $H$ the multiplication by $n \varphi$ on the right.

Let $B$ denote the group of all bijections of $S$ leaving every ker $\pi$-class invariant. Let $M$ be generated by $B$ and $N$. Then every element of $M$ induces a self-embedding of $H$ which is right multiplication by an element of $H$. This gives a homomorphism $M \rightarrow H$, which is $\varphi$ on $N$. We will show that this gives the desired $M / B \cong H$ isomorphism.

The homomorphism is clearly surjective and $B$ is mapped to the identity. So we only have to check that for $h_{1}, h_{2} \in H \backslash G$ there is a $\tau \in B$ with

$$
h_{1}^{R} \cdot h_{2}^{R} \cdot \tau=\left(h_{1} h_{2}\right)^{R}
$$

This already determines $\tau$ on some subset of $S$ and we have to show that this partial map extends to a bijection leaving the ker $\pi$-classes invariant. Since the partial map is injective, what we have to check is that, for every $h \in H$, in the class $h \pi^{-1}$ the complement of the domain respectively the image of the partial map have the same cardinality. But all these cardinalities are $\lambda$ since they obviously cannot be larger and, for instance, $\lambda \times\{h\} \times\{1\}$ is contained in both of the complements.

Now we prove (3.6). For this purpose, we define the action of $M$ on $B$. Let $m \in M$ and $b \in B$. Since $m$ is injective, it induces an isomorphism between its domain $S$ and its image $S m$. The condition $m b^{m}=b m$ is equivalent to the fact that $b^{m}$ equals $m^{-1} b m$ on $S m$. Hence we define $b^{m}$ to be $m^{-1} b m$ on $S m$ (this is an isomorphism of $S m$ ) and to be the identity on $S \backslash S m$.

It is an easy exercise to check that this definition satisfies all the requirements:

First, $b^{m}$ is clearly an isomorphism of $S$ preserving every $\operatorname{ker} \pi$-class. Moreover, $b^{m}$ is so defined that $m b^{m}=b m$ holds.

Now we check that the action of $m$ is a group homomorphism, i.e. for 
$b_{1}, b_{2} \in B$ we have $\left(b_{1} b_{2}\right)^{m}=b_{1}^{m} b_{2}^{m}$. This holds indeed, because both maps are identical outside $S m$ and restricting to $S m$ the equation becomes $m^{-1} b_{1} b_{2} m=\left(m^{-1} b_{1} m\right)\left(m^{-1} b_{2} m\right)$.

Last, we have to check that the representation of $M$ on $B$ is a homomorphism of monoids. This means that, for $b \in B$ and $m_{1}, m_{2} \in M$, we have $b^{m_{1} m_{2}}=\left(b^{m_{1}}\right)^{m_{2}}$ and $b^{1}=b$. The latter is clear from the definition.

For the former, note that $S m_{1} m_{2} \subseteq S m_{2}$. Thus outside $S m_{2}$ both $b^{m_{1} m_{2}}$ and $\left(b^{m_{1}}\right)^{m_{2}}$ are identical. They are also identical on $S m_{2} \backslash S m_{1} m_{2}=(S \backslash$ $\left.S m_{1}\right) m_{2}$, the map $b^{m_{1} m_{2}}$ by definition, and the map $\left(b^{m_{1}}\right)^{m_{2}}$ because $b^{m_{1}}$ is identical on $S \backslash S m_{1}$. Finally, let us check that that the maps $b^{m_{1} m_{2}}$ and $\left(b^{m_{1}}\right)^{m_{2}}$ coincide on $S m_{1} m_{2}$ as well. The former is $\left(m_{1} m_{2}\right)^{-1} b\left(m_{1} m_{2}\right)$ restricted to $S m_{1} m_{2}$, while the latter is $m_{2}^{-1}\left(m_{1}^{-1} b m_{1}\right) m_{2}$. These maps are obviously the same.

So far we have proved the existence of a monoid $M$ with right cancellation and a subgroup $B$ on which $M$ acts satisfying (3.6) such that $M / B \cong H$. Only the last sentence of the proposition has not been proved yet, which states that $M$ can be chosen so that $|M| \leq \lambda$. We are going to prove that there exists a submonoid $M_{0}$ of $M$ of cardinality at most $\lambda$ and a subgroup $B_{0}$ of both $B$ and $M_{0}$ such that $B_{0}$ is closed under the action of $M_{0}$ and $M_{0} / B_{0} \cong H$. This will finish the proof of the proposition.

Such an $M_{0}$ and $B_{0}$ are easy to find: for every $h_{1}, h_{2} \in H \backslash G$ choose a $\tau\left(h_{1}, h_{2}\right) \in B$ such that $h_{1}^{R} h_{2}^{R} \tau\left(h_{1}, h_{2}\right)=\left(h_{1} h_{2}\right)^{R}$. We have proved above that such $\tau\left(h_{1}, h_{2}\right)$ exists. Let $B_{0}$ be the subgroup of $B$ generated by all the $\tau\left(h_{1}, h_{2}\right)$ 's and closed under the action of $N$. Let $M_{0}$ be the submonoid of $M$ generated by $N$ and $B_{0}$. Thus $B_{0}$ is closed under the action of $M_{0}$. Recall that $|H| \leq \lambda$ by assumption and thus $\left|B_{0}\right| \leq \lambda$ and $\left|M_{0}\right| \leq \lambda$. One can show $M_{0} / B_{0} \cong H$ in the same way as we have shown $M / H \cong B$ above.

\subsection{A fully rigid system of abelian $p$-groups}

In this section we construct a fully rigid system of abelian $p$-groups, from which we will build the groups for Theorem 3.1.2 in the next section. The main statement is Corollary 3.3 .3 . 


\section{Preliminaries}

We begin with recalling some notions from the theory of abelian $p$-groups and introducing some convenient notation.

Pure subgroups and torsion-completion A subgroup $B$ of an abelian group $A$ is a pure subgroup, in notation $B \subseteq_{*} A$, if, for every integer $n$, one has $B \cap n A=n B$. For example, direct summands are pure subgroups.

Recall that a group is $\Sigma$-cyclic if it is a direct sum of cyclic groups. A basic subgroup of an abelian $p$-group $A$ is a pure $\Sigma$-cyclic subgroup $B$ such that $A / B$ is divisible. Abelian $p$-groups have many basic subgroups; for instance, every subgroup $F$ with the property $F \cap p^{n} A=0$ for some $n$ is contained in a basic subgroup of $A$. See [6, Chapter VI.] for the theory of basic subgroups.

We will usually not use the definition of pure subgroups directly. Instead we will use Lemma 3.3.1 to find pure subgroups.

Recall, for example from [7, Section 68], the following notions: An abelian group $A$ is separable if $\bigcap_{n=1}^{\infty} n A=0$. The torsion-completion $\bar{A}$ of a separable abelian $p$-group $A$ is the torsion part of the $p$-adic completion $\hat{A}$. Note that $A$ is always pure in $\hat{A}$ and $\hat{A} / A$ is divisible.

Also recall that homomorphisms from a pure dense subgroup to a torsioncomplete group extend uniquely to the whole group.

There is a nice criterion for a subgroup of a torsion-completion to be pure:

Lemma 3.3.1. Let $B$ be a separable abelian p-group and $\bar{B}$ its torsion completion. Let $C$ be a group such that $B \subseteq C \subseteq \bar{B}$.

Then $C$ is pure in $\bar{B}$ if and only if the factor group $C / B$ is divisible.

Proof. We know that $B$ is a pure subgroup of $\bar{B}$. So, by [6, Lemma 26.1], $C$ is pure in $\bar{B}$ if and only if $C / B$ is pure in $\bar{B} / B$. Since $\bar{B} / B$ is divisible, its pure subgroups are exactly the divisible ones, which finishes the proof.

Unavoidable homomorphisms As we have already mentioned, in this section we want to construct a fully rigid system of abelian $p$-groups, i.e. a system of groups such that among its members the only homomorphisms are 
the "unavoidable" ones. Now we introduce convenient notations for these homomorphisms.

If $A$ is any abelian group then there are obvious homomorphisms from $A$ to $\bigoplus_{i \in I} A e_{i}$, namely, the embeddings $e_{i}$. Of course, we can take linear combinations of the $e_{i}$ 's: we can consider $\sum_{i \in I} j_{i} e_{i}$ for any $j=\sum_{i \in I} j_{i} e_{i} \in$ $\bigoplus_{i \in I} \mathbb{Z} e_{i}$. It will be convenient to write $j$ for this homomorphism and refer to it as multiplication by $j$.

Moreover, if $A$ is an abelian $p$-group, then $\bigoplus_{i \in I} \mathbb{Z} e_{i}$ is pure in the $p$ adically complete group $\operatorname{Hom}\left(A, \bigoplus_{i \in I} A e_{i}\right)$ (see [6, Theorem 46.1]) and hence, by completion, we have multiplication by any $j=\sum_{i \in I} j_{i} e_{i} \in\left(\sum_{i \in I} J_{p} e_{i}\right)^{\wedge}$. The explicit formula is

$$
\left(\sum_{i \in I} j_{i} e_{i}\right) a:=\sum_{i \in I} j_{i} a e_{i} \quad(a \in A) .
$$

If $\bigoplus_{i \in I} A e_{i} \subseteq B$ and $D \subseteq B$ then we also use $j$ to denote the homomorphism from $A$ to $B / D$ sending $x \in A$ to $j x+D$.

A homomorphism $\phi: A \rightarrow B$ between abelian $p$-groups is small if, for every natural number $k$, there is an $n$ such that $p^{n} A\left[p^{k}\right] \phi=0$. We denote by $\operatorname{Small}(A, B)$ the group of small homomorphisms between $A$ and $B$.

There are plenty of small homomorphisms between abelian $p$-groups, for instance, the basic subgroup is always the image of a small endomorphism, see [24] or [6, Theorem 36.1].

\section{The fully rigid system}

The theorem and corollary below are the main results of this section. These form an extended version of Theorem 2.4 in [4].

Theorem 3.3.2. Let $D$ be a separable abelian p-group of cardinality at most $\lambda=\lambda^{\aleph_{0}}$. Then there are an abelian p-group $A$ containing $D$ as a pure subgroup and a system of subgroups $A_{X}(X \subseteq \lambda)$ of $A$ containing $D$ such that

(i) $A_{X} / D$ is unbounded and separable for all $X \subseteq \lambda$; 
(ii) $\left|A_{X}\right|=\lambda$ for all $X \subseteq \lambda$;

(iii) $A_{X} \subseteq A_{Y}$ iff $X \subseteq Y$, for all $X, Y \subseteq \lambda$;

(iv) for any cardinal $\tau$ :

$$
\operatorname{Hom}\left(A_{X},\left(A_{Y} / D\right)^{(\tau)}\right)=\operatorname{Small}\left(A_{X},\left(A_{Y} / D\right)^{(\tau)}\right) \oplus \begin{cases}\widehat{J_{p}^{(\tau)}}, & \text { if } X \subseteq Y \\ 0, & \text { if } X \nsubseteq Y .\end{cases}
$$

The proof of this theorem will be done in several steps using the Black Box principle. The arguments will take up the most of this section. Despite the great effort we invest in proving the theorem, we will not use it directly in later sections. We will use the theorem only through the following corollary, which we prove immediately.

Corollary 3.3.3. Let $D, \lambda$ and $A_{X}(X \subseteq \lambda)$ be as in Theorem 3.3.2. Then for any subgroup $A_{X}^{\bullet}$ of finite index in $A_{X}$

every homomorphism from $A_{X}^{\bullet}$ to a $\Sigma$-cyclic group is small,

and for any $\Sigma$-cyclic group $C$ with $D^{(\tau)} \subseteq C \subseteq \subseteq_{*} \overline{D^{(\tau)}}$ and $C \cap \bar{D}^{(\tau)}=D^{(\tau)}$ :

$$
\operatorname{Hom}\left(A_{X}^{\bullet}, A_{Y}^{(\tau)}+C\right)=\operatorname{Small}\left(A_{X}^{\bullet}, A_{Y}^{(\tau)}+C\right) \oplus \begin{cases}\widehat{J_{p}^{(\tau)}}, & \text { if } X \subseteq Y \\ 0, & \text { if } X \nsubseteq Y .\end{cases}
$$

Proof. First, it is enough to prove the corollary for $A_{X}^{\bullet}=A_{X}$ because every finite index subgroup of an abelian $p$-group contains a direct summand of finite index. Let us prove this statement as an exercise on basic subgroups. Therefore let $G$ be an abelian $p$-group and $E$ a finite index subgroup of $G$. Let $B$ be a basic subgroup of $G$, then $G /(B+E)$ is a finite divisible group and hence must be zero. Thus $G=B+E$, so $B$ has a finite direct summand $F$ such that $G=F+E$. Then $F$ is a pure subgroup of $G$ and hence $F \cap p^{n} G=p^{n} F=0$ where $p^{n}=|F|$. So $F \cap E$ is contained in a basic subgroup of $E$, and therefore it is also contained in a finite direct summand 
of $E$, i.e. $E=H \oplus L$ where $F \cap E \subseteq H$ is finite. It is easy to check that $G=(F+H) \oplus L$ and so $L \subseteq E$ is a direct summand of $G$ of finite index.

Second, every homomorphism from $A_{X}$ to a $\Sigma$-cyclic group (in particular to $C$ ) is small: It is enough to check this for countable $\Sigma$-cyclic groups since any non-small homomorphism to a $\Sigma$-cyclic group $S$ can be composed with a projection onto a countable direct summand of $S$ such that the composition is non-small. Countable $\Sigma$-cyclic groups can be embedded into $A_{X} / D$, so the statement is just an easy application of the theorem.

Third, by the theorem, it is enough to show that every homomorphism $\phi$ from $A_{X}$ to $\bigoplus_{\alpha<\tau} A_{Y}+C$ is small if $\phi$ composed by the factor map $\bigoplus_{\alpha<\tau} A_{Y}+$ $C \rightarrow\left(\bigoplus_{\alpha<\tau} A_{Y}+C\right) / C \cong \bigoplus_{\alpha<\tau} A_{Y} / D$ is small.

Finally, let $\phi: A_{X} \rightarrow \bigoplus_{\alpha<\tau} A_{Y}+C$ be a homomorphism, which is small when projected onto $\left(\bigoplus_{\alpha<\tau} A_{Y}+C\right) / C$. This means that for every $k$ there is an $n$ such that

$$
p^{n} A_{X}\left[p^{k}\right] \phi \subseteq C
$$

Now we are going to show that $\phi$ is small, and this will finish the proof. To this end, we define $\tilde{\phi}$, a modification of $\phi$, which will map all of $A_{X}$ to $C$. Let us choose a basic subgroup $B=\oplus C_{i}$ of $A_{X}$ where the $C_{i}$ 's are cyclic subgroups. We choose $\tilde{\phi}$ on these $C_{i}$ 's as any homomorphism to $C$ which coincides with $\phi$ on $C \phi^{-1} \cap C_{i}$. This is possible because $C$ is pure in $\bigoplus_{\alpha<\tau} A_{Y}+C$. So $\tilde{\phi}$ is defined on $B$, and extends uniquely to an $A_{X} \rightarrow \bar{C}$ homomorphism. Now we check that the image of $\tilde{\phi}$ is contained in $C$.

Let $k$ be a positive integer and choose $n$ as in (3.14). Then $\tilde{\phi}$ and $\phi$ agree on $p^{n} A_{X}\left[p^{k}\right]$ because they agree on its dense subgroup $p^{n} B\left[p^{k}\right]$ by construction. Since $A_{X}\left[p^{k}\right] \subseteq B+p^{n} A_{X}\left[p^{k}\right]$, the function $\tilde{\phi}$ maps $A_{X}\left[p^{k}\right]$ to $C$. This holds for all $k$, so $A_{X} \tilde{\phi} \subseteq C$.

Since any homomorphism from $A_{X}$ to a $\Sigma$-cyclic group is small, $\tilde{\phi}$ is small. Hence for any $k$ there is an $m \geq n$ ( $n$ is from (3.14) $)$ such that

$$
p^{m} A_{X}\left[p^{k}\right] \phi=p^{m} A_{X}\left[p^{k}\right] \tilde{\phi}=0
$$


Thus $\phi$ is small.

\section{Reduction to $\tau=\omega$}

Now we start proving Theorem 3.3.2. In this subsection we reduce the claim in (iv) to $\tau=\omega$ and leave the proof of the reduced theorem to the rest of this section.

In other words, we prove that if a system $A_{X}(X \subseteq \lambda)$ satisfies (iv) only for $\tau=\omega$ than it satisfies it for all cardinals $\tau$.

Recall that (iv) for $\tau=\omega$ means that

$$
\operatorname{Hom}\left(A_{X},\left(A_{Y} / D\right)^{(\omega)}\right)=\operatorname{Small}\left(A_{X},\left(A_{Y} / D\right)^{(\omega)}\right) \oplus \begin{cases}\widehat{J_{p}^{(\omega)}}, & \text { if } X \subseteq Y \\ 0, & \text { if } X \nsubseteq Y .\end{cases}
$$

Since $A_{Y} / D$ is a direct summand of $\left(A_{Y} / D\right)^{(\omega)}$, it is clear from (3.15) that for any homomorphism $\theta: A_{X} \rightarrow A_{Y}$ there is a unique $j \in J_{p}$ such that $\theta-j$ is small. Moreover, if $X \nsubseteq Y$ then $j=0$. We shall make extensive use of this observation.

Let $\tau$ be any cardinal and $\phi: A_{X} \rightarrow\left(A_{Y} / D\right)^{(\tau)}$ a homomorphism. We have to prove that there is a unique $j \in\left(J_{p}^{(\tau)}\right)^{\wedge}$ such that $\phi-j$ is small and that $j=0$ if $X \nsubseteq Y$.

For $H \subseteq \tau$ let $\pi_{H}:\left(A_{Y} / D\right)^{(\tau)} \rightarrow\left(A_{Y} / D\right)^{(H)}$ denote the canonical projection. For $\alpha \in \tau$ let $\pi_{\alpha}:=\pi_{\{\alpha\}}:\left(A_{Y} / D\right)^{(\tau)} \rightarrow A_{Y} / D$ denote the projection onto the $\alpha$ 'th summand. Then there is a unique $j_{\alpha} \in J_{p}$ such that $\phi \pi_{\alpha}-j_{\alpha}$ is small.

Obviously, $j:=\sum_{\alpha \in \tau} j_{\alpha} e_{\alpha}$ is the only possible choice for $j \in\left(J_{p}^{(\tau)}\right)^{\wedge}$, for which $\phi-j$ can be small. It is also clear that $j=0$ if $X \subseteq Y$. So it is left to prove that $j \in\left(J_{p}^{(\tau)}\right)^{\wedge}$ and $\phi-j$ is small.

Next we show that $j$ is an element of $\left(J_{p}^{(\tau)}\right)^{\wedge}$, in other words, that, for every $n$, all but finitely many of the $j_{\alpha}$ 's are divisible by $p^{n}$. Otherwise there would be a countably infinite set $H \subseteq \tau$ such that $p^{n} \nmid j_{\alpha}$ for $\alpha \in H$. Applying 3.15 to $\phi \pi_{H}$ we would get a $j^{\prime} \in\left(J_{P}^{(H)}\right)^{\wedge}$ such that $\phi \pi_{H}-j^{\prime}$ is small. Clearly $j^{\prime}=\sum_{\alpha \in H} j_{\alpha} e_{\alpha}$ contradicting that none of the $j_{\alpha}$ 's is divisible 
by $p^{n}$.

So far, letting $\psi:=\phi-j$, we know that, for any $\alpha \in \tau$, the homomorphism $\psi \pi_{\alpha}=\phi \pi_{\alpha}-j \pi_{\alpha}=\phi \pi_{\alpha}-j_{\alpha}$ is small. We are going to show that $\psi$ is small.

If $\psi$ is not small then there is a countable $H \subseteq \tau$ such that $\psi \pi_{H}$ is not small. Applying (3.15), we obtain a $k:=\sum_{\alpha \in H} k_{\alpha} e_{\alpha} \in\left(J_{p}^{(H)}\right)^{\wedge}$ such that $\psi \pi_{H}-k$ is small. Since $\psi \pi_{\alpha}$ is small for any $\alpha \in H$, this implies that $k_{\alpha}=0$. Hence $k=0$ and $\psi \pi_{H}=\psi \pi_{H}-k$ is small, a contradiction.

\section{The Black Box}

The heart of the construction is a version of the Black Box very similar to (2.3) in [4]. Also ideas from [2] are used.

Before stating the Black Box, we introduce the necessary terminology and notation.

Recall that a tree is a partially ordered set $(P,<)$ such that, for every $p \in P$, the set of all elements of $P$ smaller than $p$ is well-ordered. The order type of this set is called the height of $p$. A subtree of $P$ is a subset $Q \subseteq P$ such that whenever $p \in P, q \in Q$ and $p \leq q$, we have $p \in Q$. Note that a subtree is a tree with the induced ordering, and every element has the same height in the tree and the subtree. The elements of a tree are also called nodes.

For example, for any ordinal $\alpha$, the set of all functions $\sigma: n \rightarrow \alpha$ for all $n \in \omega$ ordered by inclusion form a tree, which we denote by ${ }^{<\omega} \alpha$. The set of all strictly monotone increasing functions $\sigma: n \rightarrow \lambda$ for all $n \in \omega$ form a subtree of ${ }^{<\omega} \lambda$, which will play a crucial role in the Black Box. We denote it by $T$.

For $\sigma: n \rightarrow \lambda$, we denote by $l(\sigma)$ the height of $\sigma$, which is clearly $n$ and coincides with the domain of $\sigma$.

A branch of a tree is a maximal linearly ordered subset of the tree. For example, the branches of ${ }^{<\omega} \alpha$ are in bijection with all the functions $f: \omega \rightarrow \alpha$, where $f$ corresponds to the branch $\{f\lceil n: n \in \omega\}$. Similarly, the branches of $T$ are in bijection with all the strictly monotone increasing functions $f: \omega \rightarrow$ $\lambda$. 
For any tree $T_{0}$, let $\mathrm{Br} T_{0}$ denote the set of branches of $T_{0}$.

As we claimed before, we are mainly interested in the tree $T$, which we now treat as a set of generators of the group $Z:=\bigoplus_{\sigma \in T} C\left(p^{l(\sigma)+1}\right) a_{\sigma}$ with fixed generators $a_{\sigma}$ for the summands. This group is not our whole universe, as we will work in the torsion-completion $\overline{D \oplus Z}$ where $D$ is a fixed separable abelian $p$-group. Since $\bar{Z}$ is canonically contained in $\prod_{\sigma \in T} C\left(p^{l(\sigma)+1}\right) a_{\sigma}$, every $x \in \overline{D \oplus Z}=\bar{D} \oplus \bar{Z}$ has coordinates in the product. Let $x_{\sigma}$ denote the $\sigma^{\prime}$ th coordinate of $x$. We define the support $[x]:=\left\{\sigma \mid x_{\sigma} \neq 0\right\}$ of $x$ as the set of nodes where it is not zero. This does not depend on the component of $x$ in $\bar{D}$, which has no significance for us. If $x=\sum_{i \in \omega} x_{i} e_{i} \in \overline{Z^{(\omega)}} \subseteq \prod_{i \in \omega} \bar{Z}$ then let the support of $x$ be $[x]:=\bigcup_{i \in \omega}\left[x_{i}\right]$, the union of the supports of the components of $x$. The support $[S]$ of a subset $S$ of $\bar{D} \oplus \bar{Z}$ or $\overline{Z^{(\omega)}}$ is simply the union of the supports of its elements. Let the norm of $S$ be

$$
\|S\|:=\min \left\{\alpha \leq \lambda \mid[S] \subseteq{ }^{<\omega} \alpha\right\}
$$

By the norm of a single node $\sigma$ we mean the norm of the one-element set $\{\sigma\}$, i.e. $\|\sigma\|:=\|\{\sigma\}\|$.

Set

$$
\begin{aligned}
V & :=D \oplus \bigoplus_{\sigma \in T} C\left(p^{l(\sigma)+1}\right) a_{\sigma}, \\
W & :=\bigoplus_{\alpha<\omega} V e_{\alpha} .
\end{aligned}
$$

Our last notion involves most of the previously defined concepts:

Definition 3.3.4. A trap is a 6-tuple $\left(f, D^{\prime}, S, P, \varphi, \gamma\right)$ where

- $f:<\omega \omega \rightarrow T$ is an embedding of trees;

- $D^{\prime}$ is a countable subgroup of $D$;

- $S$ is a countable subtree of $T$;

- $P:=D^{\prime} \oplus \bigoplus_{\sigma \in S} C\left(p^{l(\sigma)+1}\right) a_{\sigma}$;

- $\varphi: P \rightarrow \overline{\left(P / D^{\prime}\right)^{(\omega)}}$ is a homomorphism; 
- $\gamma \leq \lambda$ is an ordinal;

- $\operatorname{Im} f \subseteq S$;

- $\|\tau\|=\|S\|$ for all branches $\tau \in \operatorname{Br}(\operatorname{Im} f)$.

We are ready to formulate the Black Box.

Lemma 3.3.5 (Black Box). Let D be a separable abelian p-group of cardinality at most $\lambda=\lambda^{\aleph_{0}}$. Then there is a sequence $\left(f_{\alpha}, D_{\alpha}^{\prime}, S_{\alpha}, P_{\alpha}, \varphi_{\alpha}, \gamma_{\alpha}\right)_{\left(\alpha<\lambda^{*}\right)}$ of traps such that $\left|\lambda^{*}\right|=\lambda$ and

(i) $\beta<\alpha \Rightarrow\left\|S_{\beta}\right\| \leq\left\|S_{\alpha}\right\|$;

(ii) $\beta \neq \alpha \Rightarrow \operatorname{Br}\left(\operatorname{Im} f_{\beta}\right) \cap \operatorname{Br}\left(\operatorname{Im} f_{\alpha}\right)=\emptyset$;

(iii) $\beta+2^{\aleph_{0}} \leq \alpha \Rightarrow \operatorname{Br}\left(\operatorname{Im} f_{\alpha}\right) \cap \operatorname{Br}\left(\left[S_{\beta}\right]\right)=\emptyset$;

(iv) if $S \subseteq \bar{V}$ and $I \subseteq D$ are countable sets, $\phi: \bar{V} \rightarrow \bar{W}$ is a homomorphism and $\gamma<\lambda$, then there is an $\alpha<\lambda^{*}$ such that

$$
S \subseteq \overline{P_{\alpha}}, I \subseteq D_{\alpha}^{\prime}, \gamma_{\alpha}=\gamma \text { and } \phi \uparrow P_{\alpha}=\varphi_{\alpha}
$$

Proof. The proof is basically the same as the one of (A.7) in [2] and hence omitted. The Black Box is very robust under changing its setting.

\section{Construction of abelian $p$-groups}

In this subsection we prove Theorem 3.3 .2 by constructing suitable groups.

We use the sequence $\left(f_{\alpha}, D_{\alpha}^{\prime}, S_{\alpha}, P_{\alpha}, \varphi_{\alpha}, \gamma_{\alpha}\right)_{\left(\alpha<\lambda^{*}\right)}$ of traps produced by Lemma 3.3 .5 to construct the group $A$. We also introduce some notation: Let

$$
v^{k}(\tau):=\sum_{n \geq k} p^{n-k} a_{\tau \uparrow n} \quad(\tau \in \operatorname{Br}(T), k \in \omega)
$$

We call a sequence $\tilde{x}=\left(x^{k}: k \in \omega\right) \subseteq \bar{V}$ a $V$-chain if $x^{k}-p x^{k+1} \in V$ for all $k \in \omega$. This means that in $\bar{V} / V$ the sequence is a $p$-divisibility chain. For example, $\left(v^{k}(\tau): k \in \omega\right)$ is a $V$-chain for any $\tau \in \operatorname{Br}(T)$. 
For a $V$-chain $\tilde{x}$, a branch $\tau \in \operatorname{Br} T$ and a subgroup $B \subseteq \bar{V}$ set

$$
B(\tau, \tilde{x}):=\left\langle B, v^{k}(\tau)+x^{k} \mid k \in \omega\right\rangle .
$$

We recursively choose elements $g_{\beta}^{k}$ and define pure subgroups $A^{\alpha}$ of $\bar{V}$ for $\beta, \alpha<\lambda^{*}$ such that

(i) $A^{\alpha}:=\left\langle D \oplus Z, g_{\beta}^{k} \mid \beta<\alpha\right\rangle$.

(ii) $g_{\beta}^{k}=v^{k}\left(\tau_{\beta}\right)+x_{\beta}^{k}$ for some $\tau_{\beta} \in \operatorname{Br}\left(\operatorname{Im} f_{\beta}\right)$ and some $V$-chain $\tilde{x}_{\beta}=$ $\left(x_{\beta}^{k}\right) \subseteq \overline{P_{\beta}}$ with $o\left(x^{0}\right)=p$ and $\left\|x_{\beta}^{k}\right\|<\left\|S_{\beta}\right\|\left(=\left\|\tau_{\beta}\right\|\right)$ for all $k \in \omega$. Note that $v^{k}\left(\tau_{\beta}\right) \in \overline{P_{\beta}}$ and so $g_{\beta}^{k} \in \overline{P_{\beta}}$.

(iii) If $g_{\beta}^{k} \varphi_{\beta} \notin\left(A^{\beta+1} / D\right)^{(\omega)}$ for some $\beta<\alpha$ and $k \in \omega$ then also $g_{\beta}^{k} \varphi_{\beta} \notin$ $\left(A^{\alpha} / D\right)^{(\omega)}$.

(iv) Moreover, whenever possible, we choose $g_{\beta}^{k}$ 's in such a way that, for some $k \in \omega$,

$$
g_{\beta}^{k} \varphi_{\beta} \notin\left(A^{\beta+1} / D\right)^{(\omega)}=\left(A^{\beta}\left(\tau_{\beta}, \tilde{x}_{\beta}\right) / D\right)^{(\omega)} .
$$

Note, we shall see later that $\operatorname{such} g_{\beta}^{k}$ 's (satisfying (ii) and (iii)) exist.

Finally we put

$$
A:=\bigcup_{\alpha<\lambda^{*}} A^{\alpha}=\left\langle D \oplus Z, g_{\beta}^{k} \mid \beta<\lambda^{*}, k<\omega\right\rangle
$$

and

$$
A_{X}:=\left\langle D \oplus Z, g_{\alpha}^{k} \mid \gamma_{\alpha} \in X \cup\{\lambda\}\right\rangle \quad(X \subseteq \lambda)
$$

Properties of $A$ We are going to prove some properties of $A$.

Lemma 3.3.6. $A_{X}$ is a pure subgroup of $\bar{V}$ for every $X \subseteq \lambda$. In particular, $A=A_{\lambda}$ is a pure subgroup of $\bar{V}$. 
Proof. Since $V \subseteq A_{X} \subseteq \bar{V}$, the purity of $A_{X}$ is equivalent to the divisibility of $A_{X} / V$ by Lemma 3.3.1. But $A_{X} / V$ is divisible since it is generated by divisibility chains: $\left(g_{\beta}^{k}+V\right)_{k \in \omega}$ for all $\beta<\lambda^{*}$ with $\gamma_{\beta} \in X \cup\{\lambda\}$.

The following lemma describes how the elements of $A$ look like.

Lemma 3.3.7 (Recognition Lemma). Let $h \in A \backslash V$. Then

(a) There is a unique $\alpha<\lambda^{*}$ such that $h \in A^{\alpha+1} \backslash A^{\alpha}$.

(b) With $\alpha$ from (a), there exist unique ordinals $\alpha=\alpha(0)>\ldots>\alpha(n)$ with $\left\|S_{\alpha(i)}\right\|=\left\|S_{\alpha}\right\|$ for $i \leq n$ and there is an ordinal $\mu<\left\|S_{\alpha}\right\|$ such that

$$
[h]_{\mu}=F \cup \bigcup_{i=0}^{n}\left[\tau_{\alpha(i)}\right]_{\mu}
$$

where the union is disjoint and $F$ is a finite set and

$$
[x]_{\mu}:=\{\sigma \in[x]:\|\sigma\|>\mu\}
$$

for all $x \in \bar{V}$ or $x \subseteq T$.

In particular, $\|h\| \geq\left\|S_{\alpha}\right\|$.

(c) If $\tau$ is a branch of $T$ with $\|\tau\| \geq\left\|S_{\alpha}\right\|$ then there is an $l \in \omega$ and $z \in \mathbb{Z}$ such that

$$
h_{\sigma}=z v^{l}(\tau)_{\sigma}
$$

for all but finitely many elements $\sigma$ of $\tau$. Moreover, if $\tau \notin \operatorname{Br}\left(\operatorname{Im} f_{\alpha+1}\right)$ then $z$ can be chosen as zero, which means that $[h] \cap \tau$ is finite in this case.

Remark. Since $D \subseteq V$ and the norm and support do not depend on the component in $\bar{D}$, we have same results on $h \in(A / D) \backslash(V / D)$ (with the only change that we say $h \in\left(A^{\alpha+1} / D\right) \backslash\left(A^{\alpha} / D\right)$ in (a) $)$. It is also easily seen that the lemma generalizes to every $h \in(A / D)^{(\omega)}$. In this case we have $z \in \mathbb{Z}^{(\omega)}$ in (c). 
Proof. Condition (a) follows from the fact that the groups $A^{\alpha}\left(\alpha<\lambda^{*}\right)$ form a continuous increasing chain whose union is $A$ and which starts at $A^{0}=V$.

To see (b) and (c), let $h \in A^{\alpha+1}$. Then it can be written, by (i) of the construction and since the $g_{\beta}^{k}$, s form a $V$-chain, as

$$
h=f+\sum_{i=0}^{m} z_{i} g_{\alpha(i)}^{k}
$$

for some $f \in V, \alpha \geq \alpha(0)>\ldots>\alpha(m), k, m \in \omega$ and $z_{i} \in \mathbb{Z}$. We can assume that none of the summands are zero except maybe $f$. Since $h \notin A^{\alpha}$, we must have $\alpha=\alpha(0)$. By Lemma 3.3.5 (ii) we have, for some $n \leq m$,

$$
\left\|S_{\alpha}\right\|=\left\|S_{\alpha(0)}\right\|=\ldots=\left\|S_{\alpha(n)}\right\|>\left\|S_{\alpha(n+1)}\right\| \geq \ldots \geq\left\|S_{\alpha(m)}\right\| .
$$

We rewrite 3.25 in the form

$$
h=f+\sum_{i=1}^{n} z_{i} v^{k}\left(\tau_{\alpha(i)}\right)+\underbrace{\sum_{i=1}^{n} z_{i} x_{\alpha(i)}^{k}+\sum_{i=n+1}^{m} z_{i} g_{\alpha(i)}^{k}}_{y} .
$$

To show (b), we claim that, if $\mu<\left\|S_{\alpha}\right\|$ is large enough, then $\|y\|<\mu$ and the sets $[f]_{\mu},\left[z_{i} v^{k}\left(\tau_{\alpha(i)}\right)\right]_{\mu}=\left[v^{k}\left(\tau_{\alpha(i)}\right)\right]_{\mu}=\left[\tau_{\alpha(i)}\right]_{\mu}$ are pairwise disjoint. Then (3.23) will hold with $F:=[f]_{\mu}$ (which is finite since $[f]$ is finite).

To prove the claim, our first observation is that $\|y\|<\left\|S_{\alpha}\right\|$ because each summand of $y$ in 3.27 has norm less than $\left\|S_{\alpha}\right\|$. Since $z_{i} v^{k}\left(\tau_{\alpha(i)}\right) \neq 0$ for any $i \leq n$ by assumption, we have $\left[z_{i} v^{k}\left(\tau_{\alpha(i)}\right)\right]=\left[v^{k}\left(\tau_{\alpha(i)}\right)\right]$ and hence $\left[z_{i} v^{k}\left(\tau_{\alpha(i)}\right)\right]_{\mu}=\left[v^{k}\left(\tau_{\alpha(i)}\right)\right]_{\mu}$. Note that $\tau \backslash\left[v^{k}\left(\tau_{\alpha(i)}\right)\right]=\{\tau \uparrow 0, \ldots, \tau \uparrow k\}$, and hence $[\tau]_{\mu}=\left[v^{k}\left(\tau_{\alpha(i)}\right)\right]_{\mu}$ whenever $\mu>(k) \tau$.

For $i \neq j \leq n$ we know that $\tau_{\alpha(i)} \cap \tau_{\alpha(j)}$ is finite from condition (ii) of Lemma 3.3.5, and thus, for $\left\|\tau_{\alpha(i)} \cap \tau_{\alpha(j)}\right\|<\mu$, the sets $\left[v^{k}\left(\tau_{\alpha(i)}\right)\right]_{\mu}$ and $\left[v^{k}\left(\tau_{\alpha(j)}\right)\right]_{\mu}$ are disjoint. Moreover, since $[f]$ is finite and $\left\|S_{\alpha}\right\|$ is a limit ordinal, every element of $[f]_{\mu}$ has norm greater than $\left\|S_{\alpha}\right\|$, for $\mu$ large enough. In this case, $[f]_{\mu}$ will be disjoint from the sets $\left[v^{k}\left(\tau_{\alpha(i)}\right)\right]_{\mu}$ for $i \leq n$.

Summarizing the above, we can find a common $\mu \leq\left\|S_{\alpha}\right\|$ satisfying all 
the requirements above.

Finally, let us prove (c). In fact, it is enough to assume that $\|\tau\|>\mu$ where $\mu$ is as above. If $\tau$ differs from $\tau_{\alpha(0)}, \ldots, \tau_{\alpha(n)}$ then $\tau$ intersects $[h]_{\mu}$ in a finite set because it intersects every set on the right-hand side of (3.23) in a finite set. Since $\|\tau\|>\mu$, it follows that $\tau \cap[h]$ is finite and hence (3.24) is satisfied with $z=0$ and $l$ arbitrary.

On the other hand, if $\tau=\tau_{\alpha(i)}$ for some $i \leq n$ then 3.24 holds for $\|\sigma\|>\mu$ with $z:=z_{i}$ and $l:=k$.

Corollary 3.3.8. A contains no element from the torsion-completion of $D$ but the elements of $D$, i.e. $A \cap \bar{D}=D$. Moreover, $A_{X} / D$ is a pure subgroup of $\bar{Z}$ with basic subgroup $Z$ for every $X \subseteq \lambda$.

Proof. If $h \in A \backslash V$ then $[h] \neq \emptyset$ by Lemma 3.3.7 (b). In particular, $h \notin \bar{D}$ because elements of $\bar{D}$ have empty support. Thus

$$
A \cap \bar{D}=V \cap \bar{D}=(D+Z) \cap \bar{D}=D+\underbrace{(Z \cap \bar{D})}_{0}=D .
$$

It remains to show that $A_{X} / D$ is a pure subgroup of $\bar{Z}$ with basic subgroup $Z$. Consider the canonical projection $\pi: \overline{D \oplus Z}=\bar{D} \oplus \bar{Z} \rightarrow \bar{Z}$. Its kernel is $\bar{D}$. Hence $\pi$ restricted to $A_{X}$ has kernel ker $\pi \uparrow A_{X}=A_{X} \cap \bar{D}=D$. So $\pi$ induces an isomorphism $A_{X} / D \cong A_{X} \pi \subseteq \bar{Z}$. Now $Z \subseteq A_{X}$ and hence $Z=Z \pi \subseteq A_{X} \pi$.

So far we know that $Z \subseteq A_{X} \pi \subseteq \bar{Z}$. We will prove that $A_{X} \pi / Z$ is divisible, which implies immediately that $Z$ is a basic subgroup of $A_{X} \pi$ and, by Lemma 3.3.1, $A_{X} \pi$ is pure in $\bar{Z}$. The proof is just an easy calculation:

$$
A_{X} \pi / Z=A_{X} \pi / Z \pi \cong A_{X} /\left(\operatorname{ker}\left(\pi \uparrow A_{X}\right)+Z\right)=A_{X} /(D+Z) .
$$

The last group is divisible, again by Lemma 3.3.1, since $A_{X}$ is pure in $\overline{D \oplus Z}$.

Our final lemma provides us many small homomorphisms. 
Lemma 3.3.9. With A from (3.21), $A / D$ is a thin group, which means that every homomorphism from a torsion-complete p-group to it is small. Furthermore, $(A / D)^{(\omega)}$ is thin.

Proof. The argument is essentially the same as the proof of (8.2) in [2]. Nevertheless, it is presented here.

Since direct sums of thin groups are thin (see [23, Theorem 2]), $(A / D)^{(\omega)}$ is a thin group if $A / D$ is thin. So we have to prove the latter only.

Let $X$ be a torsion-complete group and $\psi: X \rightarrow A / D$ a homomorphism. Suppose, for contradiction, that $\psi$ is not small, i.e. there is a natural number $k$, a strictly increasing sequence of natural numbers $\left(n_{i}\right)_{i \in \omega}$ and elements $e_{i} \in p^{n_{i}} X\left[p^{k}\right]$ such that $h_{i}:=e_{i} \psi \neq 0$.

Without loss of generality, we may assume (by passing to subsequences) that

$$
\left\|h_{i}\right\| \leq\left\|h_{i+1}\right\| \quad \text { for } i \in \omega
$$

and that there are nodes $\sigma_{i} \in\left[h_{i}\right]$ such that:

(A) $\left\|\sigma_{i}\right\| \leq\left\|\sigma_{i+1}\right\|$ for $i \in \omega$;

(B) $\sup \left\|\sigma_{i}\right\|=\sup \left\|h_{i}\right\|$;

(C) $p^{l\left(\sigma_{i}\right)} \mid h_{j}$ if $i<j$;

(D) if infinitely many $\sigma_{i}$ lie on the same branch then all of the $\sigma_{i}$ 's do.

For any sequence $\left(\varepsilon_{i}\right) \subseteq\{0,1\}^{\omega}$, the sum $z:=\sum \varepsilon_{i} e_{i}$ is convergent and

$$
h=\sum \varepsilon_{i} h_{i}=z \psi \in A / D .
$$

Note that $\sigma_{i} \notin\left[h_{j}\right]$ for $i<j$ by (C), so the $\sigma_{i}{ }^{\prime}$ th coordinate of $h$ is the same as that of $\sum_{j \leq i} \varepsilon_{j} h_{j}$, which we write as $z_{i} a_{\sigma_{i}}$ for some $z_{i} \in \mathbb{Z}$. So we can choose the $\varepsilon_{i}$ 's recursively such that $h_{\sigma_{i}}$ differs from any preassigned value. 
This way we can achieve that

$$
h_{\sigma_{i}} \neq \begin{cases}0 & \text { if } i \text { is even } \\ z_{i-1} p^{l\left(\sigma_{i}\right)-l\left(\sigma_{i-1}\right)} a_{\sigma_{i}} & \text { if } i \text { is odd }\end{cases}
$$

This implies with the help of (B) and (3.29) that $\sup \left\|\sigma_{i}\right\|=\|h\|$. By Lemma 3.3.7 (b), we have, for some $\alpha(0)>\ldots>\alpha(n)$ and $\mu<\|h\|$,

$$
[h]_{\mu}=F \cup \bigcup_{i=0}^{n}\left[\tau_{\alpha(i)}\right]_{\mu}
$$

where the union is disjoint and $F$ is a finite set. Recall that

$$
[x]_{\mu}:=\{\sigma \in[x]:\|\sigma\|>\mu\} .
$$

Thus the finitely many branches $\tau_{\alpha(0)}, \ldots, \tau_{\alpha(n)}$ cover almost all of the $\sigma_{i}$ 's, and hence infinitely many of them lie on the same branch. By $(\mathrm{D})$, then all the $\sigma_{i}$ 's lie on the same branch $\tau$. So, by Lemma 3.3.7 (c), for all but finitely many of the $\sigma_{i}$ 's we have $h_{\sigma_{i}}=\left(z v^{k}(\tau)\right)_{\sigma_{i}}$ contradicting 3.30 for almost all odd $i$.

Existence of the $g_{\alpha}^{k}$ 's Next we show that the $g_{\alpha}^{k}$ 's needed for the construction exist. Recall that the $g_{\alpha}^{k}$ 's are defined recursively. The next lemma shows that the construction carries over at every step.

Lemma 3.3.10. Suppose that for some $\alpha<\lambda^{*}$, there are $g_{\beta}^{k}(\beta<\alpha)$ satisfying condition (iii) of the construction.

Then there is a branch $\tau \in \operatorname{Br}\left(\operatorname{Im} f_{\alpha}\right)$ such that, for any $V$-chain $\tilde{x}=$ $\left(x^{k}: k<\omega\right) \subseteq \bar{V}$ with $o\left(x^{0}\right)=p$ and $\left\|x^{k}\right\|<\left\|S_{\alpha}\right\|$ for all $k$, we have

$$
g_{\beta}^{k} \varphi_{\beta} \notin\left(A^{\alpha}(\tau, \tilde{x}) / D\right)^{(\omega)}
$$

for all $\beta<\alpha$ and $k \in \omega$ with $g_{\beta}^{k} \varphi_{\beta} \notin\left(A^{\alpha} / D\right)^{(\omega)}$.

Proof. First we show that, for a fixed $\beta<\alpha$, there is at most one branch $\tau$ for which 3.32 fails for some $\tilde{x}$. 
Suppose for contradiction that there are two branches $\tau_{1}$ and $\tau_{2}$ of $\operatorname{Im} f_{\alpha}$, and sequences $\tilde{x}_{1}$ and $\tilde{x}_{2}$ such that

$$
g_{\beta}^{k} \varphi_{\beta} \in\left(A^{\alpha}\left(\tau_{i}, \tilde{x}_{i}\right) / D\right)^{(\omega)} \quad(i=1,2) .
$$

Then there are $z_{i} \in \mathbb{Z}^{(\omega)}(i=1,2)$ and $l \in \omega$ with

$$
g_{\beta}^{k} \varphi_{\beta}-z_{i}\left(v^{l}\left(\tau_{i}\right)+x_{i}^{l}\right) \in\left(A^{\alpha} / D\right)^{(\omega)} \quad(i=1,2) .
$$

Subtracting the equations gives

$$
c:=z_{1} v^{l}\left(\tau_{1}\right)-z_{2} v^{l}\left(\tau_{2}\right)+\left(z_{1} x_{1}^{l}-z_{2} x_{2}^{l}\right) \in\left(A^{\alpha} / D\right)^{(\omega)} .
$$

The support of both the second summand and the expression in brackets intersect the branch $\tau_{1}$ in a finite set. The former because $\tau_{1} \cap \tau_{2}$ is finite, the branches being distinct, the latter because it has norm less than $\left\|S_{\alpha}\right\|$. Thus $[c] \cap \tau_{1}$ is infinite unless $p^{l}$ divides $z_{1}$. But Lemma 3.3.7 (b) prevents the former. The latter is also impossible since then 3.34 would reduce to $g_{\beta}^{k} \varphi_{\beta} \in\left(A^{\alpha} / D\right)^{(\omega)}$ in case $i=1$. Therefore we have a contradiction in both cases and thus there can only be at most one branch $\tau$ failing 3.32 .

Second, we show that, if $g_{\beta}^{k} \varphi_{\beta} \in\left(A^{\alpha}(\tau, \tilde{x}) / D\right)^{(\omega)}$ for some $\beta<\alpha, k \in \omega$ and a sequence $\tilde{x}$, then $\tau \in \operatorname{Br}\left(S_{\beta}\right)$. This implies that $\tau \in \operatorname{Br}\left(S_{\beta}\right) \cap \operatorname{Br}\left(\operatorname{Im} f_{\alpha}\right)$ and hence $\beta+2^{\aleph_{0}}>\alpha$ by Lemma 3.3.5 (iii).

As before, $g_{\beta}^{k} \varphi_{\beta} \in\left(A^{\alpha}(\tau, \tilde{x}) / D\right)^{(\omega)}$ means that, for some $l \in \omega$ and $z \in$ $\mathbb{Z}^{(\omega)}$,

$$
g_{\beta}^{k} \varphi_{\beta}-z\left(v^{l}(\tau)+x^{l}\right) \in\left(A^{\alpha} / D\right)^{(\omega)} .
$$

The support of this element must intersect $\tau$ in a finite set by Lemma 3.3.7 (a). Now $\left[z v^{l}(\tau)\right]$ intersects $\tau$ in an infinite set but $\left[z x^{l}\right]$ in a finite set. So $\left[g_{\beta}^{k} \varphi_{\beta}\right] \cap \tau$ must be infinite. Since $\left[g_{\beta}^{k} \varphi_{\beta}\right] \subseteq S_{\beta}$, we obtain that the subtree $S_{\beta}$ contains infinitely many elements of $\tau$ and hence must contain all of $\tau$. Thus $\tau \in \operatorname{Br}\left(S_{\beta}\right)$.

Now we estimate the number of the branches $\tau$ in $\operatorname{Br}\left(\operatorname{Im} f_{\alpha}\right)$ which do not 
satisfy 3.32 for some $\beta<\alpha$ and $k \in \omega$. For every $\beta<\alpha$ there is at most one $\tau$, so the number of $\tau$ 's is less than or equal to the number of $\beta$ 's. Let $\beta_{0}$ be the smallest among the $\beta$ 's. Then $\alpha<\beta_{0}+2^{\aleph_{0}}$. Since all the $\beta$ 's lie between $\beta_{0}$ and $\alpha$ the number of $\beta$ 's is less than continuum.

All in all, there are less than continuum many branches $\tau \in \operatorname{Br}\left(\operatorname{Im} f_{\alpha}\right)$ which fail the lemma. Since $\operatorname{Br}\left(\operatorname{Im} f_{\alpha}\right)$ consists of continuum many elements, it must have an element satisfying the lemma.

The homomorphisms Now we turn to the proof of equation iv of Theorem 3.3 .2 for $\tau=\omega$, namely that, for every $X, Y \subseteq \lambda$,

$$
\operatorname{Hom}\left(A_{X},\left(A_{Y} / D\right)^{(\omega)}\right)=\widehat{J_{p}^{(\omega)}} \oplus \begin{cases}0 & \text { if } X \nsubseteq Y \\ \operatorname{Small}\left(A_{X},\left(A_{Y} / D\right)^{(\omega)}\right) & \text { if } X \subseteq Y .\end{cases}
$$

The key for the proof is the following:

Proposition 3.3.11. Let $\phi: A_{X} \rightarrow(A / D)^{(\omega)}$ be a homomorphism $(X \subseteq \lambda)$. Then there exists $j \in\left(J_{p}^{(\omega)}\right)^{\wedge}$ such that $\phi-j$ is small.

Now we can easily deduce (3.37) from the proposition.

Proof of (3.37). Observe that since $A_{Y} \subseteq A$ we have $\left(A_{Y} / D\right)^{(\omega)} \subseteq(A / D)^{(\omega)}$. Hence, for any $\phi: A_{X} \rightarrow\left(A_{Y} / D\right)^{(\omega)}$ (viewed as a map to $\left.(A / D)^{(\omega)}\right)$, there is a $j \in J_{p}^{(\omega)}$ such that $\phi-j$ is small by the proposition. Now we show that if $X \nsubseteq Y$ then $j=0$ and hence $\phi$ is small. We have to be careful because we do not even know that the image of $\phi-j$ is contained in $\left(A_{Y} / D\right)^{(\omega)}$.

Suppose for contradiction that $X \nsubseteq Y$ and $j \neq 0$. So there are $\gamma \in X \backslash Y$ and a largest $l \in \omega$ such that $p^{l}$ divides $j$. By Lemma 3.3.5, there is an $\alpha<\lambda^{*}$ such that $\gamma=\gamma_{\alpha}$. Since $\phi$ is small, we can choose an $n$ such that $p^{n} A_{X}\left[p^{l+1}\right](\phi-j)=0$. Hence $p^{n} g_{\alpha}^{n+l} \phi=p^{n} g_{\alpha}^{n+l} j \notin\left(A_{Y} / D\right)^{(\omega)}$, a contradiction.

Finally, the sum on the right-hand side of (3.37) is direct because if $0 \neq j \in J_{p}^{(\omega)}$ then $j$ is not a small homomorphism. In fact, the kernel of $j$ is $A_{X}\left[p^{k}\right]$ where $k$ is the largest natural number such that $p^{k}$ divides $j$. Since 
$A_{X}$ is unbounded, we have $p^{n} A_{X}\left[p^{k+1}\right] \nsubseteq A_{X}\left[p^{k}\right]=\operatorname{ker} j$, for every $n \in \omega$, showing that $j$ is not small.

It remains to prove Proposition 3.3.11, which we prepare now. In the proof, we shall consider the (unique) extension of $\phi$ to a homomorphism $\overline{D \oplus Z} \rightarrow \overline{Z^{(\omega)}}$, which we also denote by $\phi$. This extension exists because $\phi$ is automatically continuous in the $p$-adic topology.

We gain control over $\phi$ by the following lemma. This is the only place in the proof where we use the special choice of $g_{\alpha}^{k}$ 's.

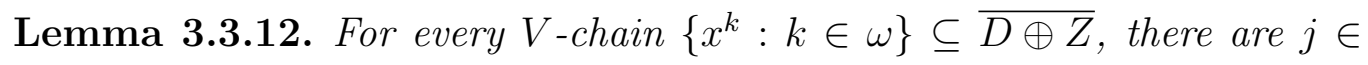
$\mathbb{Z}^{(\omega)}$ and $l \in \mathbb{N}$ such that $x \phi-j x^{l} \in(A / D)^{(\omega)}$ where $x=x^{0}$.

Recall that for $j=\sum j_{k} e_{k} \in \mathbb{Z}^{(\omega)}$ and $x \in A$ we use the notation $j x:=$ $\sum j_{k} x e_{k} \in(A / D)^{(\omega)}$.

Proof. By (iv) of Lemma 3.3.5, there is an $\alpha<\lambda^{*}$ such that

- $\gamma_{\alpha}=\lambda\left(\right.$ so $g_{\alpha}^{k} \in A_{X}$ for all $\left.k \in \omega\right)$,

- $\left\|x^{k}\right\|,\left\|x^{k} \phi\right\|<\left\|S_{\alpha}\right\|$ for all $k \in \omega$,

- $\left[x^{k}\right] \cup\left[x^{k} \phi\right] \subseteq S_{\alpha}$ for all $k \in \omega$,

- $\phi \uparrow P_{\alpha}=\varphi_{\alpha}$.

Recall from Lemma 3.3 .10 that there is a branch $\tau \in \operatorname{Br}\left(\operatorname{Im} f_{\alpha}\right)$ such that, for any $V$-chain $\tilde{y}=\left(y^{l}: l \in \omega\right) \subseteq \overline{P_{\alpha}}$,

$$
g_{\beta}^{k} \varphi_{\beta} \notin\left(A^{\alpha}(\tau, \tilde{y}) / D\right)^{(\omega)}
$$

whenever $g_{\beta}^{k} \varphi_{\beta} \notin\left(A^{\alpha} / D\right)^{(\omega)}$ for some $\beta<\alpha$ and $k \in \omega$, i.e. whenever $g_{\beta}^{k} \varphi_{\beta} \notin\left(A^{\beta+1} / D\right)^{(\omega)}$ by (iii) of the construction.

However, $g_{\alpha}^{k} \varphi_{\alpha}=g_{\alpha}^{k} \phi \in(A / D)^{(\omega)}$ and hence $g_{\alpha}^{k} \phi_{\alpha} \in\left(A^{\alpha+1} / D\right)^{(\omega)}$, for all $k \in \omega$. By (iv) of the construction, we must have, for all $k \in \omega$ and $V$-chain $\tilde{y}$ with $o\left(y^{0}\right)=p$

$$
\left(v^{k}(\tau)+y^{k}\right) \varphi_{\alpha} \in\left(A^{\alpha}(\tau, \tilde{y}) / D\right)^{(\omega)} .
$$


Let $o(x)=p^{k+1}$. We apply 3.39$)$ to the $V$-chains $\left(y^{k}=0 \mid k \in \omega\right)$ and the one defined by $y^{0}=p^{k} x, y^{1}=p^{k-1} x, \ldots, y^{k}=x=x^{0}$ and $y^{k+l}=x^{l}$ for all $l \in \omega:$

$$
\begin{aligned}
v^{k}(\tau) \varphi_{\alpha} & \in\left(\left\langle A^{\alpha}, v^{l}(\tau) \mid l \in \omega\right\rangle / D\right)^{(\omega)} \\
\left(v^{k}(\tau)+x\right) \varphi_{\alpha} & \in\left(\left\langle A^{\alpha}, v^{k+l}(\tau)+x^{l} \mid l \in \omega\right\rangle / D\right)^{(\omega)} .
\end{aligned}
$$

Note that $\left\langle A^{\alpha}, v^{k+l}(\tau)+x^{l} \mid l \in \omega\right\rangle=\left\langle A^{\alpha}, v^{0}+p^{k} x, \ldots, v^{k-1}(\tau)+p x, v^{k+l}(\tau)+\right.$ $x^{l}|l \in \omega\rangle$ because $V \subseteq A^{\alpha}$. Now we deduce from the above that, for some $l \in \omega$ and $j, m \in \mathbb{Z}^{(\omega)}$,

$$
\begin{aligned}
v^{k}(\tau) \varphi_{\alpha}-j v^{k+l}(\tau) & \in\left(A^{\alpha} / D\right)^{(\omega)} \\
\left(v^{k}(\tau)+x\right) \varphi_{\alpha}-m\left(v^{k+l}(\tau)+x^{l}\right) & \in\left(A^{\alpha} / D\right)^{(\omega)} .
\end{aligned}
$$

Subtracting (3.42) from 3.43 and using that $x \varphi_{\alpha}=x \varphi$ we obtain:

$$
b:=\left(x \varphi-m x^{l}\right)-(m-j) v^{k+l}(\tau) \in\left(A^{\alpha} / D\right)^{(\omega)} .
$$

The first expression in brackets has norm less than $\left\|S_{\alpha}\right\|$ by the choice of $\alpha$. Moreover, due to Lemma 3.3.7 (c), $[b] \cap \tau$ is finite, which is only possible for $(m-j) v^{k+l}(\tau)=0$. So 3.44 reduces to $x \varphi-m x^{l} \in\left(A^{\alpha} / D\right)^{(\omega)}$ as required.

Finally, we prove our proposition.

Proof of Proposition 3.3.11. Let $\phi: A_{X} \rightarrow(A / D)^{(\omega)}$ be a homomorphism. Recall that $\phi$ extends to a homomorphism $\overline{D \oplus Z} \rightarrow \overline{Z^{(\omega)}}$, which we also denote by $\phi$.

It follows from Lemma 3.3 .12 that $\phi$ maps $\bar{D}+A$ into $(A / D)^{(\omega)}$. For if $x \in \bar{D}$ then we can choose a $V$-chain $\left(x^{k}: k \in \omega\right)$ from $\bar{D}$ with $x=x^{0}$, since $\bar{D}$ is pure in $\bar{V}$. By the preceding lemma, there are $k \in \omega$ and $j \in \mathbb{Z}^{(\omega)}$ such that $x \phi-j x^{k} \in(A / D)^{(\omega)}$. But since $x^{k} \in \bar{D}$, automatically $j x^{k}=0$, so $x \phi \in(A / D)^{(\omega)}$. One can similarly show that $x \phi \in(A / D)^{(\omega)}$ if $x \in A$. 
Hence $\phi$ induces a homomorphism $\tilde{\phi}: \bar{V} /(\bar{D}+A) \cong \bar{Z} /(A / D)=: Q \rightarrow$ $\bar{W} /(A / D)^{(\omega)}$. The next step is determining this induced homomorphism. (It is interesting that we look at $\phi$ where it was originally not defined.)

Again by Lemma 3.3.12, each $x \in Q$ is mapped by $\tilde{\phi}$ to some $j x^{k}$ for some $j \in \mathbb{Z}^{(\omega)}$. Here $x^{k} \in Q$ and $p^{k} x^{k}=x$. Note that we must have $o(x) j x^{k}=o(x) x \tilde{\phi}=0$ where $o(x)$ is the order of $x$. This implies that $p^{k}$ divides $j$, say $j=m p^{k}$, so in fact $x \tilde{\phi}=m p^{k} x^{k}=m x$.

So we have proved that for every $x \in Q$ there is a $j \in\left(J_{p}^{(\omega)}\right)^{\wedge}$ such that $x \tilde{\phi}=j x$. In particular, $\tilde{\phi}$ maps $Q$ to $Q^{(\omega)}$. Now a usual argument on completeness, which we present in the next two paragraphs, shows that we can find a $j \in\left(J_{p}^{(\omega)}\right)^{\wedge}$ such that $x \tilde{\phi}=j x$ for all $x \in Q$.

For a fixed $x \in Q$ the $j$ 's for which $x \tilde{\phi}=j x$ form a coset of $o(x)\left(J_{p}^{(\omega)}\right)^{\Upsilon}$. Any two of these cosets are either disjoint or one is contained in the other. In fact, we will prove that there are no disjoint ones among them, so the cosets form a chain. By completeness, their intersection is non-empty, which is what we claimed.

So let us prove that, for any $x, y \in Q$, there is a $j \in\left(J_{p}^{(\omega)}\right)^{\wedge}$ such that $x \tilde{\phi}=j x$ and $y \tilde{\phi}=j y$. By the fundamental theorem of abelian groups, the group $\langle x, y\rangle$ is a direct sum of two cyclic groups: $\langle x, y\rangle=\langle a\rangle \oplus\langle b\rangle$ where the summands may be trivial. Let $p^{k}=o(a) \geq o(b)=p^{l}$. There are $j, m$ and $t \in\left(J_{p}^{(\omega)}\right)^{\wedge}$ such that

$$
a \tilde{\phi}=j a \quad b \tilde{\phi}=m b \quad(a+b) \tilde{\phi}=t(a+b) .
$$

Since $(a+b) \tilde{\phi}=a \tilde{\phi}+b \tilde{\phi}$ we thus get $j a+m b=t a+t b$. But $a$ and $b$ are independent, so we must have that $p^{k}$ divides $j-t$ and $p^{l}$ divides $m-t$. So $p^{l}$ divides $j-m$ and hence $b \tilde{\phi}=m b=j b$. Thus $\tilde{\phi}$ coincides with $j$ on $\langle a, b\rangle=\langle x, y\rangle$ as claimed.

So $\tilde{\phi}=j$ for some $j \in J_{p}^{(\omega)}$. Returning to the homomorphism $\phi$, this means that $\psi:=\phi-j$ maps the whole $\bar{V}$ to $(A / D)^{(\omega)}$. By Lemma 3.3.9, $(A / D)^{(\omega)}$ is a thin group. Hence $\psi$ must be small by the definition of thin groups, which completes the proof. 


\subsection{Construction of locally finite $p$-groups}

Now we construct the groups $P_{X}$ and $G_{X}$ for $X \subseteq \kappa$ to prove Theorem 3.1.2.

We fix a continuous cofinal sequence $\left(\beta_{\alpha}: \alpha<\operatorname{cf} \kappa\right)$ in $\kappa$ such that $\beta_{0}=0$ and set $\beta_{\mathrm{cf} \kappa}:=\kappa$. We will construct a smooth chain of groups $\left(G_{X \cap \beta_{\alpha}}^{\alpha}\right)_{\alpha \leq \operatorname{cf} \kappa}$ for every $X \subseteq \kappa$ and set $G_{X}=G_{X}^{\text {cf } \kappa}$. For $\alpha<\operatorname{cf} \kappa$ the group $G_{X}^{\alpha}$ will have cardinality less than $\kappa$. It will be convenient to use $G:=G_{\kappa}$ for the group containing all of the groups we are working with. For each $\alpha$ the collection of groups $\left(G_{X}^{\alpha}\right)_{X \subseteq \beta_{\alpha}}$ will satisfy a similar but significantly weaker condition than required by the theorem: First, we need monoids $M_{X}^{\alpha}$ with right cancellation generated by $M$ and $G_{X}^{\alpha}$. The monoid $M_{X}^{\alpha}$ is designed to be the submonoid $M \cdot \operatorname{Inn} G_{X}^{\alpha}$ of $\operatorname{Emb} G$. We require that $M_{X}^{\alpha}$ acts on every $G_{X}^{\alpha}$ by monomorphisms. $G_{X}^{\alpha}$ acts by inner automorphisms. These actions should be compatible with each other for all $\alpha$ and $X$.

To simplify notation we put $G^{\alpha}:=G_{\beta_{\alpha}}^{\alpha}$ and $M^{\alpha}:=M_{\beta_{\alpha}}^{\alpha}$. We require

(i) $G_{X}^{\gamma} \cap G^{\alpha}=G_{X \cap \beta_{\alpha}}^{\alpha}$ for all $X \subseteq \beta_{\gamma}$ and $\alpha<\gamma$;

(ii) $G_{X}^{\alpha} \subseteq G_{Y}^{\beta}$ iff $X \subseteq Y$ and $\alpha \leq \beta$;

(iii) $M_{X}^{\alpha} \subseteq M_{Y}^{\beta}$ iff $X \subseteq Y$ and $\alpha \leq \beta$;

(iv) if $g \in G_{X}^{\alpha}, m \in M$ and $g^{m} \in B$ then $g \in B$ for all $\alpha$ and $X$;

(v) $\operatorname{Emb}\left(G_{X}^{\alpha}, G_{Y}^{\alpha}\right) \cap M \cdot \operatorname{Inn} G^{\alpha}= \begin{cases}M \cdot \operatorname{Inn} G_{Y}^{\alpha} & \text { if } X \subseteq Y, \\ \emptyset & \text { if } X \nsubseteq Y .\end{cases}$

So far this is easy to establish by a recursive definition, which is our next task. 


\section{Initial step}

For $\alpha=0$ we set

$$
\begin{aligned}
A & :=\bigoplus_{n<\omega} \mathbb{Z}_{p^{n}}, \\
P & :=\bigoplus_{x \in M} A^{(\lambda)} e_{x}, \\
G^{0} & :=P \rtimes B .
\end{aligned}
$$

Here $B$ acts on $P$ by the right regular representation of the coordinates $e_{x}$.

Let $M$ act on $G^{0}$ by moving the coordinates in $\bigoplus_{x \in M} A^{(\lambda)} e_{x}$ by the right regular representation and mapping $b \in B$ to $b^{m}$ by $m$. Clearly $M \cap \operatorname{Inn} G^{0}=$ $B$.

\section{The monoids $M_{X}^{\alpha}$ 's}

Semidirect products of semigroups Recall that if $N$ and $T$ are semigroups and $N$ is acting on $T$ then their semidirect product $N \ltimes T$ is a semigroup with underlying set $N \times T$ and multiplication given by the rule

$$
\left(n_{1}, t_{1}\right) \cdot\left(n_{2}, t_{2}\right)=\left(n_{1} \cdot n_{2}, t_{1}^{n_{2}} t_{2}\right) \quad\left(n_{1}, n_{2} \in N, t_{1}, t_{2} \in T\right)
$$

where $t^{n}$ is the action of $n$ on $t$.

Definition of $M_{X}^{\alpha}$ Once $G_{X}^{\alpha}$ is given with the action of $M$ on it, we define $M_{X}^{\alpha}$ as a factor of a semi-direct product by a congruence $\sim$ :

$$
\begin{gathered}
(m b, g) \sim(m, b g) \quad(b \in B, m \in M, b \in B) \\
M_{X}^{\alpha}:=M \ltimes G_{X}^{\alpha} / \sim
\end{gathered}
$$

with the given action of $M$ on $G_{X}^{\alpha}$. Requirement (iii) then follows from (ii).

Note that right cancellation holds in $M_{X}^{\alpha}$ because of (iv). In details: let 
$\left(m_{1}, g_{1}\right),\left(m_{2}, g_{2}\right)$ and $(m, g)$ be elements of $M_{X}^{\alpha}$ and suppose

$$
\left(m_{1}, g_{1}\right)(m, g)=\left(m_{2}, g_{2}\right)(m, g)
$$

This means that

$$
\left(m_{1} m, g_{1}^{m} g\right)=\left(m_{2} m, g_{2}^{m} g\right)
$$

so that for some $b \in B$

$$
m_{1} m b=m_{2} m, \quad b g_{2}^{m} g=g_{1}^{m} g .
$$

The right equation gives us $b=\left(g_{1} g_{2}^{-1}\right)^{m} \in B$, so by (iv) we have

$$
c:=g_{1} g_{2}^{-1} \in B \text {. }
$$

Thus $b=c^{m}$. Substituting this into the left equation of (3.54) and applying (3.5):

$$
m_{2} m=m_{1} m c^{m}=m_{1} c m .
$$

Since right cancellation holds in $M$, we get $m_{2}=m_{1} c$. From (3.55) we also get $g_{1}=c g_{2}$. Hence $\left(m_{1}, g_{1}\right)=\left(m_{2}, g_{2}\right)$.

\section{Limit step}

If $\alpha$ is a limit ordinal, we simply put

$$
G_{X}^{\alpha}:=\bigcup_{\gamma<\alpha} G_{X \cap \beta_{\gamma}}^{\gamma} \quad(X \subseteq \alpha)
$$

This satisfies all the requirements: the key is that (ii) takes care that nothing can go wrong with (ii), (v) and (ii), while (iv) is automatically inherited from the smaller groups. 


\section{Successor step}

Once for some $\alpha$ the $G_{X}^{\alpha}$ 's are given we define the $G_{X}^{\alpha+1}$,s. Therefore we choose a separable abelian $p$-group $A^{\alpha}$ of cardinality $\lambda$ and consider

$$
S:=\bigoplus_{v \in M^{\alpha}}\left(A^{\alpha} \oplus A^{\left(\beta_{\alpha+1}\right)}\right) e_{v} .
$$

We define the action of $M^{\alpha}$ on $S$ by

$$
\left(\sum_{v \in M^{\alpha}} a_{v} e_{v}\right)^{g}:=\sum_{v \in M^{\alpha}} a_{v} e_{v g} \quad\left(g \in M^{\alpha}\right) .
$$

For all $X \subseteq \beta_{\alpha+1}$ and $Y \subseteq \beta_{\alpha}$ we choose subgroups $F_{X}^{\alpha}$ and $T_{Y}^{\alpha}$ of $S$ such that

$$
\begin{array}{ll}
T_{Y}^{\alpha} \subseteq \bigoplus_{v \in G^{\alpha}} A^{\alpha} e_{v} & \left(Y \subseteq \beta_{\alpha}\right), \\
F_{X}^{\alpha}:=T_{X \cap \beta_{\alpha}}^{\alpha} \oplus \bigoplus_{v \in G_{X \cap \beta_{\alpha}}^{\alpha}} A^{(X)} e_{v} & \left(X \subseteq \beta_{\alpha+1}\right) .
\end{array}
$$

These subgroups will be invariant under $M$ and conjugation by $G_{X}^{\alpha}$ such that $F_{X}^{\alpha} \subseteq F_{Y}^{\alpha}$, respectively $T_{X}^{\alpha} \subseteq T_{Y}^{\alpha}$ if and only if $X \subseteq Y$. Set

$$
G_{X}^{\alpha+1}:=F_{X}^{\alpha} \rtimes G_{X \cap \beta_{\alpha}}^{\alpha}
$$

Then the above requirements for the $G_{X}^{\alpha+1}$ 's are clearly satisfied.

Note that this definition implies that for all $x \in G \backslash G^{\alpha}$ the centralizer $C_{G^{\alpha}}(x)$ is finite: For all such $x$ there is a $\beta \geq \alpha$ with $x \in G^{\beta+1} \backslash G^{\beta}$. By 3.62 ,,$G^{\beta+1}=F^{\beta} \rtimes G^{\beta}$, so we can write $x=f g$ for some $1 \neq f \in F^{\beta}$ and $g \in G^{\beta}$. If $b \in G^{\alpha} \subseteq G^{\beta}$ stabilizes $x$ then it also stabilizes $f$ and $g$. Hence $C_{G^{\alpha}}(x) \subseteq C_{G^{\beta}}(f)$ and the latter group is also finite.

The existence of $P_{X}$ 's Also note that the above construction automatically ensures the existence of $P_{X}$ 's required by the theorem. In fact, we can define $P_{X}^{\alpha}$ 's for each $\alpha$ and $X \subseteq \beta_{\alpha}$ such that $G_{X}^{\alpha}=P_{X}^{\alpha} \rtimes B$ and $P_{X}^{\alpha} \subseteq P_{Y}^{\alpha}$ 
iff $X \subseteq Y$. The definition is

$$
P_{X}^{\alpha}:=\left\langle P, F_{X \cap \beta_{\gamma+1}}^{\gamma} \mid \gamma<\alpha\right\rangle
$$

One can prove by an easy induction on $\alpha$ that $P_{X}^{\alpha}$ is a locally finite $p$ group. The relevant equations for the induction are

$$
\begin{aligned}
P_{\emptyset}^{0} & =P, \\
P_{X}^{\alpha+1} & =F_{X \cap \beta_{\alpha}}^{\alpha} \rtimes P_{X \cap \beta_{\alpha}}^{\alpha}, \\
P^{\alpha} & =\bigcup_{\gamma<\alpha} P_{X \cap \beta_{\gamma}}^{\gamma} .
\end{aligned}
$$

The choice of $T_{X}^{\alpha}$ 's It remains to show that $\operatorname{Emb}\left(G_{X}, G_{Y}\right) \subseteq M \cdot \operatorname{Inn} G_{Y}$. This requires some care when choosing the $A^{\alpha}$ 's and $T_{X}^{\alpha}$ 's, which we now describe.

Apply Corollary 3.3 .3 to $D:=A^{(\lambda)}$. Then especially we get a collection $\left(A^{\alpha}: \alpha<\kappa\right)$ of abelian $p$-groups of cardinality $\lambda$ such that for all $\beta, \gamma$ and any subgroup $A^{\beta \bullet}$ of finite index in $A^{\beta}$ and any $\Sigma$-cyclic group $C$ and cardinal $\tau$ as in 3.13

$$
\operatorname{Hom}\left(A^{\beta \bullet}, \bigoplus_{\alpha<\tau} A^{\gamma}+C\right)=\operatorname{Small}\left(A^{\beta \bullet}, \bigoplus_{\alpha<\tau} A^{\gamma}+C\right) \oplus \delta_{\beta, \gamma} \widehat{\bigoplus} \widehat{\bigoplus}_{\alpha<\tau} J_{p}
$$

because $\lambda$ has $2^{\lambda}(\geq \kappa)$ pairwise non-comparable subsets.

For $X \subseteq \beta_{\alpha}$, we choose a $\Sigma$-cyclic group $C_{X}^{\alpha}$ which will prevent unwanted embeddings of $G_{X}^{\alpha}$, and set

$$
T_{X}^{\alpha}:=\bigoplus_{v \in G^{\alpha}} A^{\alpha} e_{v}+C_{X}^{\alpha}
$$

This will ensure that every homomorphism from $A^{\alpha}$ to $F_{X}^{\gamma}$ is small if $\alpha \neq \gamma$, and is, up to a small homomorphism, multiplication by an element from $\left(J_{p}^{\left(G^{\alpha}\right)}\right)^{\wedge}$ if $\alpha=\gamma$. 
The choice of $C_{X}^{\alpha}$ 's It does not matter how $C_{X}^{\alpha}$ is chosen for $\operatorname{cf} \alpha \neq \omega$. (One can set $C_{X}^{\alpha}:=D^{\left(G^{\alpha}\right)}$.) So let $\operatorname{cf} \alpha=\omega$. From now on, we fix $\alpha$ and construct the $C_{X}^{\alpha}$ 's.

To this end, first we choose a countable subgroup $Y$ of $G_{\emptyset}^{\alpha}$ separating $M^{\alpha}$, which means that if $m_{1}, m_{2} \in M^{\alpha}$ and $m_{1} \neq m_{2}$ then there is a $y \in Y$ such that $y m_{1} \neq y m_{2}$. This is the point where it is important that the cofinality of $\alpha$ is countable and $C_{X}^{\alpha}$ is $\Sigma$-cyclic.

The separating group $Y$ Choose a cofinal sequence $\left(\alpha_{n}: n<\omega\right)$ of ordinals in $\alpha$.

Lemma 3.4.1. There is a countable group $Y \leq G_{\emptyset}^{\alpha}$ such that the following holds.

(i) If $Y^{\bullet} \leq Y,\left[Y: Y^{\bullet}\right]<\infty$ and $h_{1} \neq h_{2} \in M^{\alpha}$ then the action of $h_{1}$ and $h_{2}$ differs on $Y^{\bullet}$ (i.e. there is an $y \in Y^{\bullet}$ with $y h_{1} \neq y h_{2}$ ).

(ii) For all $n$ there are infinitely many $x \in Y$ such that if $h_{1}, h_{2} \in M^{\alpha_{n}}$ and $x h_{1}=x h_{2}$ then $h_{1}=h_{2}$.

Proof. Let $H_{n}$ be a countably infinite subgroup of $A^{\alpha_{n}}$ and set

$$
Y:=\left\langle H_{n} e_{1}^{n} \mid n<\omega\right\rangle
$$

Obviously, if $Y^{\bullet} \leq Y,\left[Y: Y^{\bullet}\right]<\infty$ is a subgroup of finite index then $Y^{\bullet} \cap H_{n} e_{1}^{n} \neq 1$ for all $n$. Hence (ii) follows.

Let $b \in H_{n} \backslash\{1\}$. Then $b e_{1}^{n}$ satisfies (ii) for $n$. This construction also provides infinitely many such elements $b$.

Construction of $C_{X}^{\alpha} \quad$ Now we are ready to construct the $C_{X}^{\alpha}$ 's. Let $\mathcal{F}$ be a set of countable subgroups $K$ of $G^{\alpha}$ of cardinality $\left|G^{\alpha}\right|$ with $Y \leqslant K$, where $Y$ comes from Lemma 3.4.1, such that $\bigcup \mathcal{F}=G^{\alpha}$. We identify $D^{\left(G^{\alpha}\right)}$ with $\bigoplus_{K \in \mathcal{F}, x \in K}\left\langle a_{K, x}\right\rangle$ where each order $o\left(a_{K, x}\right)$ is a power of $p$ and for every $K$ and $n$ there are at most finitely many elements $x$ such that $o\left(a_{K, x}\right) \leq p^{n}$. This can be done such that $o\left(a_{K, x}\right)$ is independent of $K$ for $x \in Y$. Thus 
the direct sum contains every $C\left(p^{n}\right)$ as a direct summand $\left|G^{\alpha}\right|$ many times, which ensures that it is isomorphic to $D^{\left(G^{\alpha}\right)}$.

We choose integers $z_{K, x}$ such that $o\left(z_{K, x} a_{K, x}\right)=p$ and define the subgroup $C$ of $\overline{\bigoplus_{v \in G^{\alpha}} D e_{v}}$ and the $C_{X}^{\alpha}$ 's by

$$
\begin{aligned}
m_{K, n} & :=\sum_{\substack{x \in K \\
p^{n} \mid z_{K, x}}} p^{-n} z_{K, x} a_{K, x} e_{x}, \\
C & :=\left\langle\bigoplus_{v \in G^{\alpha}} D e_{v}, m_{K, n} \phi \mid K \in \mathcal{F}, \phi \in M^{\alpha}, n \in \mathbb{N}\right\rangle, \\
C_{X}^{\alpha} & :=\left\langle D e_{v}, m_{K, n} \phi \mid K \subseteq G_{X}^{\alpha}, v \in G_{X}^{\alpha}, \phi \in M_{X}^{\alpha}\right\rangle \leq C \quad(X \subseteq \alpha) .
\end{aligned}
$$

For every $w=\sum z_{K, x, v} a_{K, x} e_{v} \in C$, we define the support of $w$ as

$$
\operatorname{supp} w:=\left\{(K, x, v) \mid x \in K \in \mathcal{F}, v \in G^{\alpha}, z_{K, x, v} a_{K, x} \neq 0\right\} .
$$

Properties of $C_{X}^{\alpha}$. We prove some lemmas about $C_{X}^{\alpha}$ needed later.

The following lemma shows that $C$ and hence the $C_{X}^{\alpha}$ 's are $\Sigma$-cyclic.

Lemma 3.4.2. The group $C$ constructed above is $\Sigma$-cyclic.

In the proof we use, without proof, the following characterization of $\Sigma$ cyclic groups due to Kulikov, see [6, p. 87, Theorem 17.1.].

Lemma 3.4.3. [6] An abelian p-group $C$ is $\Sigma$-cyclic if and only if it is the union of an ascending chain of subgroups $\left\{C_{n}\right\}_{n<\omega}$ such that for every natural number $n$ the heights of the elements of $C_{n}$ in $C$ are bounded.

Proof of Lemma 3.4.2. Set

$$
C_{n}:=\left\langle a_{K, x} e_{v}, m_{K, n} \phi \mid o\left(a_{K, x}\right) \leq p^{n}, \phi \in M^{\alpha_{n}}, v \in G^{\alpha}, x \in K, K \in \mathcal{F}\right\rangle .
$$

Obviously, $\left\{C_{n}\right\}_{n<\omega}$ is an ascending chain of subgroups of $C$ and $C=$ $\bigcup_{n<\omega} C_{n}$. We shall prove that this chain satisfies Lemma 3.4.3.

Therefore fix a natural number $n$. Choose $x \in Y$ to satisfy Lemma 3.4.1(ii) and $o\left(a_{K, x}\right)=: k>n$ for all $K \in \mathcal{F}$. Recall that $o\left(a_{K, x}\right)$ does not depend on 
$K$ for $x \in Y$. Let

$$
w=\sum_{i=1}^{m} s_{i} m_{K_{i}, n} \phi_{i}+f, \quad f \in \bigoplus\left\langle a_{K, y}\right\rangle e_{v}, s_{i} \in \mathbb{Z}
$$

be any non-zero element of $C_{n}$. If some $a_{K, y} e_{v}$ has non-zero coefficient in $w$, then the height of $w$ is less than the exponent of $a_{K, y}$. If $m=0$ then we can clearly find such an $a_{K, y}$ with order at most $p^{n}$. If $m>0$ then $K=K_{1}$ and $y=x$ are a good choice. Anyway, the height of $w$ is less than $k$.

Lemma 3.4.4. Let $w \in C_{X}^{\alpha}, K \in \mathcal{F}$. Suppose $\phi: K \rightarrow G_{X}^{\alpha}$ is a group homomorphism and for some $v \in G_{X}^{\alpha}$

$$
\left\{a_{K, x} e_{v(x \phi)} \mid x \in K\right\} \subseteq \operatorname{supp} w
$$

Then $K \subseteq G_{X}^{\alpha}$ and $\phi$ is the restriction of some $m \in M_{X}^{\alpha}$.

We apply a well-known theorem of B. H. Neumann in the proof.

Lemma 3.4.5. [21] Let $G$ be a group, and let $G_{1}, \ldots, G_{r}$ be subgroups of $G$. If $G$ is a set union of a finite number of cosets of the $G_{i}$ irredundantly then $G_{1} \cap \cdots \cap G_{r}$ is of finite index in $G$.

Proof of Lemma 3.4.4. Write $w$ in the form

$$
w=\sum_{i=1}^{m} s_{i} m_{K_{i}, n_{i}} m_{i}+f
$$

where $f$ is in $\bigoplus\left\langle a_{K, x}\right\rangle e_{v}$ and $\left(K_{i}, m_{i}\right) \in \mathcal{F} \times M_{X}^{\alpha}$ are distinct.

Since supp $f$ is finite, replacing $w$ by $w-f$, we may assume that $f=0$.

We can write $w$ in the following form using the definition of $m_{K_{i}, n_{i}}$ :

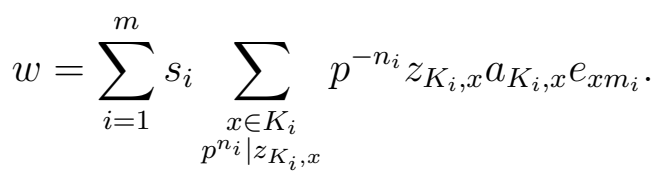

For the support of $w$ the following holds by (3.71) and (3.73):

$$
\left\{a_{K, x} e_{v(x \phi)} \mid x \in K\right\} \subseteq \operatorname{supp} w \subseteq\left\{a_{K_{i}, x} e_{x m_{i}} \mid x \in K_{i}, 1 \leq i \leq n\right\} .
$$


We define subgroups $G_{i}$ of $K$ by

$$
G_{i}:=\left\{x \in K \mid x \phi=x m_{i}\right\}
$$

We note that

$$
L_{i}:=\left\{x \in K \mid v(x \phi)=x m_{i}\right\}
$$

is either empty or a left coset of $G_{i}$. With this notation 3.74 can be expressed as

$$
K \doteq \bigcup\left\{L_{i} \mid L_{i} \neq \emptyset, K=K_{i}\right\}
$$

From now on we consider only those indices $i$ for which $K=K_{i}$. We are going to prove that for some $i$ we have $K=G_{i}$ and hence $K=K_{i} \subseteq G_{X}^{\alpha}$ and $\phi$ is the restriction of $m_{i}$. This will follow from Lemma 3.4.5 applied to the covering of $K$ above (one may add finitely many cosets of the trivial group to really obtain a covering), once we show that at most one $G_{i}$ can have finite index in $K$.

Suppose for contradiction that for some $i \neq j$ both of $G_{i}$ and $G_{j}$ have finite index. Then $K_{i}=K=K_{j}$ and also $m_{i}=m_{j}$, by Lemma 3.4.1 (i), since $m_{i}$ and $m_{j}$ coincide on the finite index subgroup $Y \cap G_{i} \cap G_{j}$ of $Y$. This contradicts the choice of the $\left(K_{i}, m_{i}\right)$ 's.

\section{Determining $\operatorname{Emb}\left(G_{X}, G_{Y}\right)$}

Now we are able to finish the proof of Theorem 3.1.2. So far, the groups $P_{X}$ $(X \subseteq \kappa)$ are constructed such that (1) holds. We know that these groups are locally finite $p$-groups of cardinality $\kappa$.

Let $X, Y \subseteq \kappa$ and $\phi \in \operatorname{Emb}\left(G_{X}, G_{Y}\right)$. We want to show that $\phi \in M^{\mathrm{cf} \kappa}=$ $M \cdot \operatorname{Inn} G$. Together with (V) at the beginning of this section, this will prove (2) and (3) of Theorem 3.1.2, which finishes the proof of the theorem. 
To simplify notation we introduce

$$
\begin{array}{rlr}
G_{X}^{\alpha} & :=G_{X \cap \beta_{\alpha}}^{\alpha}, & \\
C_{X}^{\alpha} & :=C_{X \cap \beta_{\alpha}}^{\alpha}, & \\
F_{X}^{\alpha} & :=F_{X \cap \beta_{\alpha+1}}^{\alpha} . &
\end{array}
$$

Note that this extends our previous notations from 3.57), 3.62, 3.69) and (3.61).

$G_{X}$ has two cf $\kappa$-filtrations: $G_{X}^{\alpha}(\alpha<\operatorname{cf} \kappa)$ and $G_{Y}^{\alpha} \phi^{-1}(\alpha<\operatorname{cf} \kappa)$. They coincide on a cub, compare with [5, Section 4.12]:

$$
C:=\left\{\alpha<\operatorname{cf} \kappa \mid G_{X}^{\alpha}=G_{Y}^{\alpha} \phi^{-1}\right\}
$$

Its intersection with the following stationary set is cofinal:

$$
E:=\{\alpha<\operatorname{cf} \kappa: \operatorname{cf}(\alpha)=\omega\}
$$

Let $\alpha \in E \cap C$ and $L:=F_{X}^{\alpha} \phi$. Then $L \cap G^{\alpha}=1$, so for all $y \in L \backslash\{0\}$ there is a $\gamma \geq \alpha$ such that $y \in G^{\gamma+1} \backslash G^{\gamma}$.

We claim that this $\gamma \geq \alpha$ is the same for all $y$, i.e. $L \subseteq G^{\gamma+1} \backslash G^{\gamma}$. Suppose for contradiction that $y \in G^{\mu+1} \backslash G^{\mu} \cap L$ and $z \in G^{\nu+1} \backslash G^{\nu} \cap L$ for some $\mu>\nu \geq \alpha$. Then $z^{G_{X}^{\alpha} \phi} \subseteq C_{G^{\mu}}(y)$, since $L$ is commutative, which is impossible since $\left|z^{G_{X}^{\alpha} \phi}\right|=\lambda$.

Next we show that $L^{\bullet}:=L \cap F_{X}^{\gamma}$ has finite index in $L$.

Any non-trivial element of $L$ can be written as $f a$ where $f \in F^{\gamma} \backslash\{0\}$ and $a \in G^{\gamma}$. Then for all $b \in G_{X}^{\alpha} \phi$ the conjugate $(f a)^{b}$ belongs to $L$ hence $f a$ and $(f a)^{b}$ commutes. This means

$$
f=f^{b} \cdot f^{a^{-b}} \cdot f^{-b a^{-1}} \text { and }\left[a^{b}, a\right]=1 .
$$

Note that $f$ is a non-zero element of $F_{X}^{\gamma}$, which is contained in the $p$-adic completion of $\bigoplus_{v \in G^{\alpha}} A^{\alpha} e_{v} \oplus \bigoplus_{v \in G_{X \cap \beta_{\alpha}}^{\alpha}} A^{(X)} e_{v}$ by 3.60 and 3.61. Hence there is a coordinate of $f$ not divisible by $p^{n}$ for some $n$. However, there can be only finitely many coordinates which are not divisible by $p^{n}$, say $e_{v_{1}}, \ldots, e_{v_{k}}$. 
Thus there are only finitely many $b$, namely, $v_{i}^{-1} v_{1}$ for $1 \leq i \leq k$, such that the $v_{1}$ 'th coordinate of $f^{b}$ is not divisible by $p^{n}$. Now by (3.77), the $v_{1}$ 'th coordinate of $f^{b}, f^{a^{-b}}$ or $f^{-b a^{-1}}$ is not divisible by $p^{n}$. For all but finitely many $b$ this can only be $f^{a^{-b}}$. Hence $a^{-b}$ can take only finitely many values when $b$ runs through $G_{X}^{\alpha} \phi$, i.e. $\left[G_{X}^{\alpha} \phi: C_{G_{X}^{\alpha} \phi}(a)\right]<\infty$.

Thus for $\lambda$ many elements $b$ of $G_{X}^{\alpha} \phi$ we have $a^{b}=a$. Then, on the one hand, $f^{b} \cdot f^{-1}=(f a)^{b} \cdot(f a)^{-1} \in L^{\bullet}$. Since $C_{G^{y}}(f)$ is finite, $f^{b} \cdot f^{-1} \neq 0$ for some $b$, hence $L^{\bullet} \neq 1$. On the other hand, (3.77) says that $f \cdot f^{-a^{-1}}$ commutes with these $b$ 's and hence has infinite centralizer in $G^{\gamma}$. So $f \cdot f^{-a^{-1}}=0$, i.e. $[f, a]=1$.

Now let $0 \neq x \in L^{\bullet}$. Then $x f a \in L$. As we have seen in the previous paragraph, $a$ commutes with both $f$ and $x f$. Thus $a$ commutes with $x$. This means that $L \pi \leq C_{G^{y}}(x)$ where $\pi: G^{\gamma+1}=F^{\gamma} \rtimes G^{\gamma} \rightarrow G^{\gamma}$ is the canonical projection. Hence $L \pi$ is finite, so $L^{\bullet}=\operatorname{ker} \pi$ has finite index in $L$.

So $A^{\alpha} e_{1}:=A^{\alpha} e_{1} \cap L^{\bullet}$ has finite index in $A^{\alpha} e_{1}$. Thus, by Corollary 3.3.3, the homomorphism $\phi: A^{\alpha \bullet} e_{1} \rightarrow F_{Y}^{\gamma} \subseteq F^{\gamma}$ can be expressed as

$$
\left(x e_{1}\right) \phi=\sum_{v \in G_{Y}^{\gamma}} j_{v} x e_{v}+x \sigma \quad\left(x \in A^{\alpha \bullet}\right)
$$

where $\sigma: A^{\alpha} \rightarrow F_{Y}^{\gamma}$ is a small homomorphism and $\left(j_{v}\right)_{v \in G_{Y}^{\gamma}} \subseteq\left(J_{p}^{\left(G_{Y}^{\gamma}\right)}\right)^{\wedge}$ is a collection such that for all $n$ all but finitely many of the $j_{v}$ 's are divisible by $p^{n}$. Of course, all the $j_{v}$ 's are zero if $\gamma \neq \alpha$.

Choose $n$ large enough such that $p^{n} A^{\alpha}[p] \sigma=0$ and $p^{n} A^{\alpha} e_{1} \subseteq L^{\bullet}$. 
For $K \in \mathcal{F}, K \subseteq G_{X}^{\alpha}$ consider

$$
\begin{aligned}
0 & \neq\left(p^{n} m_{K, n}\right) \phi=\left(\sum_{\substack{x \in K \\
p^{n} \mid z_{K, x}}}\left(z_{K, x} a_{K, x} e_{1}\right)^{x}\right) \phi=\sum_{\substack{x \in K \\
p^{n} \mid z_{K, x}}}\left(\left(z_{K, x} a_{K, x} e_{1}\right) \phi\right)^{x \phi} \\
& =\sum_{\substack{x \in K \\
p^{n} \mid z_{K, x}}}\left(\sum_{v \in G_{Y}^{\alpha}} j_{v} z_{K, x} a_{K, x} e_{v}\right) \\
& =\sum_{\substack{x \in K \\
p^{n} \mid z_{K, x}}} \sum_{v \in G_{Y}^{\alpha}} j_{v} z_{K, x} a_{K, x} e_{v(x \phi)} \in F_{Y}^{\alpha} \cap \overline{\bigoplus_{v \in G_{Y}^{\alpha}} D e_{v}}=C_{Y}^{\alpha} .
\end{aligned}
$$

Here $D$ is to be considered as a subgroup of $A^{\alpha}$ and $\bigoplus_{v \in G_{Y}^{\alpha}} D e_{v}$ as a subgroup of $\bigoplus_{v \in G_{Y}^{\alpha}} A^{\alpha} e_{v} \subseteq F_{Y}^{\alpha}$ in the same way as in 3.61.

There must be a $v \in G_{Y}^{\alpha}$ such that $p \nmid j_{v}$ (otherwise $\left(p^{n} m_{K, n}\right) \phi=0$ ), so $\gamma=\alpha$. For such an element $v$ we have

$$
\left\{a_{K, x} e_{v(x \phi)} \mid x \in K\right\} \subseteq \operatorname{supp}\left(p^{n} m_{K, n}\right) \phi .
$$

Then by Lemma 3.4 .4 we have $\phi \uparrow K=m$ for some $m \in M^{\alpha}$.

For different $K$ 's we get the same $m \in M$ because $m$ is uniquely determined by $\phi \uparrow Y$, see Lemma 3.4.1 (ii). Thus $\phi=m$ follows from $\bigcup \mathcal{F}=G^{\alpha}$.

So far we have established for all $\alpha \in E \cap C$ that $\phi$ acts as an $m_{\alpha} \in M^{\alpha}$ on $G_{X}^{\alpha}$.

If $\alpha<\beta$ are both in $E \cap C$ then $m_{\beta}$ has to map $G_{X}^{\alpha}$ into $G_{Y}^{\alpha}$. But if $m_{\beta} \notin M^{\alpha}$ this cannot happen since then $m_{\beta}=m \cdot g \cdot f$ for some $m \in M$ and $g \in G^{\gamma}$ and $f \in F_{\beta_{\gamma}}^{\gamma} \backslash\{1\}$ for some $\gamma \geq \alpha$ and so $f$ would be centralized by the infinite subgroup $\left(G_{X}^{\alpha} m\right)^{g}$ of $G^{\gamma}$.

Since $G_{X}^{\alpha}$ separates $M^{\alpha}$, we must have $m_{\beta}=m_{\alpha}$. Hence all of the $m_{\alpha}$ 's coincide with some $m \in M^{\text {cf } \kappa}$ when $\alpha \in E \cap C$. Thus $\phi=m$ as required. 


\section{Bibliography}

[1] Gábor Braun and Rüdiger Göbel, Outer automorphisms of locally finite p-groups, (to be published in J. Algebra), 2003.

[2] A. L. S. Corner and Rüdiger Göbel, Prescribing endomorphism algebras, a unified treatment, Proc. London Math. Soc. (3) 50 (1985), no. 3, 447479. MR 86h:16031

[3] Manfred Droste, Michèle Giraudet, and Rüdiger Göbel, All groups are outer automorphism groups of simple groups, J. London Math. Soc. (2) 64 (2001), no. 3, 565-575. MR 2002h:20050

[4] Manfred Dugas and Rüdiger Göbel, On locally finite p-groups and a problem of Philip Hall's, J. Algebra 159 (1993), no. 1, 115-138. MR 94g:20054

[5] Paul C. Eklof and Alan H. Mekler, Almost free modules, revised ed., North-Holland Mathematical Library, vol. 65, North-Holland Publishing Co., Amsterdam, 2002, Set-theoretic methods. MR 1914985

[6] László Fuchs, Infinite abelian groups. Vol. I, Pure and Applied Mathematics, Vol. 36, Academic Press, New York, 1970. MR 41 \#333

[7] _ Infinite abelian groups. Vol. II, Academic Press, New York, 1973, Pure and Applied Mathematics. Vol. 36-II. MR 50 \#2362

[8] Wolfgang Gaschütz, Nichtabelsche p-Gruppen besitzen äussere pAutomorphismen, J. Algebra 4 (1966), 1-2. MR 33 \#1365 
[9] Rüdiger Göbel and Agnes T. Paras, Automorphisms of metabelian groups with trivial center, Illinois J. Math. 42 (1998), no. 2, 333-346. MR 99d:20057

[10] _ Realizing automorphism groups of metabelian groups, Abelian groups and modules (Dublin, 1998), Trends Math., Birkhäuser, Basel, 1999, pp. 309-317. MR 2001c:20079

[11] _ Outer automorphism groups of metabelian groups, J. Pure Appl. Algebra 149 (2000), no. 3, 251-266. MR 2001d:20034

[12] P. Hall, Some constructions for locally finite groups, J. London Math. Soc. 34 (1959), 305-319. MR 29 \#149

[13] Ken Hickin, Complete universal locally finite groups, Trans. Amer. Math. Soc. 239 (1978), 213-227. MR 58 \#902

[14] C. H. Houghton, On the automorphism groups of certain wreath products, Publ. Math. Debrecen 9 (1962), 307-313. MR 27 \#215

[15] Otto H. Kegel and Bertram A. F. Wehrfritz, Locally finite groups, NorthHolland Publishing Co., Amsterdam, 1973, North-Holland Mathematical Library, Vol. 3. MR 57 \#9848

[16] Felix Leinen, Existentially closed locally finite p-groups, J. Algebra 103 (1986), no. 1, 160-183. MR 88b:20004

[17]__ A uniform way to control chief series in finite p-groups and to construct the countable algebraically closed locally finite p-groups, J. London Math. Soc. (2) 33 (1986), no. 2, 260-270. MR 87j:20039

[18] Berthold J. Maier, Existenziell abgeschlossene lokal endliche p-Gruppen, Arch. Math. (Basel) 37 (1981), no. 2, 113-128. MR 83e:20005

[19] Takao Matumoto, Any group is represented by an outer automorphism group, Hiroshima Math. J. 19 (1989), no. 1, 209-219. MR 90g:20051 
[20] Federico Menegazzo and Stewart E. Stonehewer, On the automorphism group of a nilpotent p-group, J. London Math. Soc. (2) 31 (1985), no. 2, 272-276. MR 87c:20072

[21] Bernhard Hermann Neumann, Groups covered by finitely many cosets, Publ. Math. Debrecen 3 (1954), 227-242 (1955). MR 17,234f

[22] Orazio Puglisi, A note on the automorphism group of a locally finite pgroup, Bull. London Math. Soc. 24 (1992), no. 5, 437-441. MR 93i:20043

[23] Fred Richman, Thin abelian p-groups, Pacific J. Math. 27 (1968), 599606. MR 39 \#308

[24] Tibor Szele, On the basic subgroups of abelian p-groups, Acta Math. Acad. Sci. Hungar. 5 (1954), 129-141. MR 16,9d

[25] Simon Thomas, Complete existentially closed locally finite groups, Arch. Math. (Basel) 44 (1985), no. 2, 97-109. MR 86h:03064

[26] Alexandre Zalesskiu,, A nilpotent p-group possesses an outer automorphism, Dokl. Akad. Nauk SSSR 196 (1971), 751-754. MR 43 \#350

[27] _ An example of a nilpotent group without torsion that has no outer automorphisms, Mat. Zametki 11 (1972), 21-26. MR 45 \#385 